\title{
Política Monetaria y Tasas de Interés: Experiencia Reciente para el Caso de México
}

\author{
Alejandro Díaz de León y Laura Greenham ${ }^{1}$
}

Diciembre 2000

Documento de Investigación No. 2000-08

Dirección General de Investigación Económica

BANCO DE MÉXICO

1 Se agradecen los comentarios de Moisés Schwartz Rosenthal y Alberto Torres García, así como la
asistencia de Francisco Rodríguez en la elaboración de este documento. Los autores trabajan en
Banco de México en la Dirección de Análisis Macroeconómico. Las opiniones contenidas en este
trabajo corresponden exclusivamente a los autores y no necesariamente representan el punto de
vista del Banco de México. Direcciones electrónicas: lgreen@banxico.org.mx y
adiazl@banxico.org.mx. 


\title{
Política Monetaria y Tasas de Interés: Experiencia Reciente para el Caso de México
}

\author{
Alejandro Díaz de León y Laura Greenham
}

Diciembre 2000

Documento de Investigación No. 2000-08

\begin{abstract}
Resumen
El propósito de este trabajo es analizar la efectividad de la politica monetaria para afectar la estructura de tasas de interés. En primer lugar, se presentan las variaciones en la estructura de tasas de interés en fechas posteriores a cambios en la postura de politica monetaria durante 1998, 1999 y los primeros cuatro meses de 2000. No obstante, debido a que las tasas de interés reaccionan ante diversos factores y acontecimientos, no se puede atribuir su comportamiento exclusivamente a las decisiones de política monetaria. Por ello, es necesario descomponer el efecto de un cambio en la política monetaria en dos componentes, uno de "reacción" y otro "activo". Es decir, se pretende analizar si después de una restricción monetaria, el aumento en las tasas de interés se debe exclusivamente al movimiento en la variable de politica monetaria (política monetaria "activa"), o si su comportamiento responde a eventos previamente descontados por el mercado (politica monetaria de "reacción"). Utilizando la técnica econométrica de vectores autorregresivos se presenta evidencia que sustenta el hecho de que en los últimos años, la política monetaria en México ha tenido un componente "activo".
\end{abstract}


Indice

I Introducción.

II Política monetaria en México - experiencia reciente.

II. 1 Instrumentos de política monetaria.

II.2 El uso del objetivo de saldos acumulados como instrumento de política monetaria.

II.2.1 Restricciones en la postura de política monetaria, "cortos", durante 1998.

II.2.2 Restricciones en la postura de política monetaria, "cortos", durante 1999.

II.2.3 Restricciones en la postura de política monetaria, "cortos", durante 2000 (enero-abril).

III Efecto de la política monetaria sobre las tasas de interés.

III. 1 Descomposición de la variable de política monetaria en sus componentes de "reacción" y "activo".

III.2 Análisis Empírico.

III.2.1 Resultado de los VARs sin incluir las variables de "reacción".

III.2.2 Resultado de los VARs incluyendo las variables de "reacción".

IV Conclusiones. 


\section{Introducción.}

La experiencia internacional así como la literatura económica, señalan que la mayor contribución que un banco central puede hacer al crecimiento económico radica en orientar la instrumentación de su política monetaria hacia el abatimiento de la inflación ${ }^{2}$. A este respecto, el Banco de México es, a partir de 1994, una institución autónoma cuyo objetivo prioritario es procurar la estabilidad del poder adquisitivo de la moneda nacional ${ }^{3}$.

Sin embargo, la política monetaria no actúa directamente sobre la inflación. Las decisiones de política monetaria buscan modificar tanto las expectativas de los agentes económicos, como las tasas de interés y el tipo de cambio. Esto a su vez incide sobre la demanda agregada, lo cual en su conjunto determina el comportamiento futuro de la inflación. A lo anterior se le conoce como mecanismo de transmisión de política monetaria.

El contar con bajas tasas de inflación depende, entre otros factores, de la efectividad de las acciones de política monetaria para influir en los mercados financieros, lo cual constituye el primer eslabón del mecanismo de transmisión de política monetaria. Dichas acciones las realiza el banco central a través del uso de un instrumento de política monetaria4.

Actualmente, el Banco de México utiliza como instrumento de política monetaria para afectar las tasas de interés, la determinación diaria de un objetivo para el saldo acumulado de las cuentas corrientes de la banca en el Instituto Central ${ }^{5}$. El anuncio de dicho objetivo tiene como finalidad mandar una señal sobre la postura monetaria del Banco de México. Así, un objetivo de saldos acumulados negativo o "corto" implica una restricción monetaria que se traduce en una señal de incremento en las tasas de interés para el mercado de dinero; un objetivo de saldos acumulados igual a cero implica una postura neutral y un objetivo de saldos acumulado positivo o "largo" es indicativo de un relajamiento monetario, lo cual implica una señal de disminución en las tasas de interés.

El Banco de México tiene el compromiso de restringir su postura monetaria cuando se presenten circunstancias, externas o internas, que pongan en riesgo el cumplimiento de las metas de inflación, como podrían ser un cambio desfavorable en las expectativas de inflación o la presencia de presiones en los mercados financieros 6 . Es importante mencionar que al materializarse algunos de estos eventos, los mercados inducirían por sí solos aumentos en las tasas de interés, los cuales se presentarian en adición al efecto que sobre dichas tasas tenga un ajuste en la postura de política monetaria. Por el contrario, si dichos eventos no han sido descontados por el mercado ni han dado lugar a un ajuste en las tasas de interés a satisfacción del Banco Central, sería de esperarse que tras

\footnotetext{
2 Ver Pérez López y Schwartz (1999) y Martínez (1998).

3 Banco de México, Ley del Banco de México, Diciembre 1993.

4 Se denomina instrumento de política monetaria a una variable sobre la cual un banco central tiene control directo.

${ }^{5}$ La conducción de la política monetaria por parte del Banco de México, así como el uso del objetivo de saldos acumulados se explica detalladamente en la sección II.

6 Ver Programa Monetario para 2000, Banco de México.
} 
el anuncio de una restricción monetaria se presentaran incrementos en las tasas de interés.

De esta manera, un cambio en la postura de política monetaria se puede descomponer en dos efectos: 1) cuando el Banco de México reacciona ante acontecimientos que han dado lugar por sí mismos a incrementos en las tasas de interés, a lo que llamaremos efecto "reacción", y 2) cuando el Banco de México decide modificar su postura monetaria ante eventos o circunstancias que no han sido descontados por los mercados a satisfacción del Instituto Central, a lo que denominaremos politica monetaria "preventiva" o "activa".

No obstante, sería poco probable que un cambio en el objetivo de saldos acumulados se debiera únicamente al efecto "reacción" o a la política monetaria "activa". Es de esperarse que las modificaciones en la postura de política monetaria presenten ambos componentes. De acuerdo a las circunstancias, uno de los dos tendería a dominar. Es decir, aún cuando las tasas de interés hubieran sufrido incrementos en respuesta a acontecimientos observables y descontados por los mercados, el ajuste en la postura monetaria induciría aumentos adicionales en dichas tasas. Por su parte, el anuncio de un cambio en la postura de política monetaria en respuesta a presiones inflacionarias no descontadas por el mercado a satisfacción del Instituto Central, ocasionaría además de un incremento en las tasas de interés, una reacción simultánea en otras variables de mercado, lo que a su vez tendría un impacto adicional en las tasas de interés.

Por ejemplo, la moratoria rusa en 1998 o la incertidumbre cambiaria de Brasil en 1999, ocasionaron una depreciación abrupta del tipo de cambio nacional y un incremento en las tasas de interés en México. Aunado a lo anterior, el Banco de México restringió su postura monetaria (ampliando el "corto") con el propósito de reforzar el aumento en las tasas de interés y propiciar condiciones ordenadas en el mercado cambiario. El caso anterior es un ejemplo en el cual sería de esperarse que el efecto "reacción" domine el movimiento en las tasas de interés, ya que el "corto" vino a reforzar movimientos en dichas tasas producto del propio ajuste del mercado. Por su parte, en algunas ocasiones la autoridad monetaria puede tener una percepción distinta sobre la evolución futura de la inflación (como sucedió en enero de 2000), o bien buscar una meta de inflación menor que la esperada por el mercado, ante lo cual puede elegir restringir su postura monetaria. Lo anterior sería un ejemplo en donde se esperaría que dominara el componente "activo" de la política monetaria.

Con base en lo anterior es válido preguntarse: una vez descontado el efecto "reacción", ¿sigue el cambio en el objetivo de saldos acumulados afectando a las tasas de interés?, es decir, ¿es la política monetaria en México "activa” y afecta a las tasas de interés por encima del efecto "reacción"? El presente estudio pretende contribuir a dar respuesta a estas preguntas. El objetivo de este trabajo es analizar el impacto de cambios en el objetivo de saldos acumulados, en particular la adopción o ampliación de un "corto", sobre la estructura de tasas de interés, descontando el efecto "reacción" sobre dichas tasas. Es decir, se busca analizar la respuesta de las tasas de interés, en sus diferentes plazos, ante cambios en el componente "activo" del instrumento de política monetaria.

En la sección II se describe la forma en la que el Banco de México conduce la política monetaria en la actualidad. Esta sección incluye una explicación 
detallada del régimen de saldos acumulados y del uso del "corto" como instrumento de política monetaria. En particular, se presenta un análisis estático del impacto de cada "corto", adoptado durante 1998, 1999 y los primeros cuatro meses de 2000, sobre la estructura de tasas de interés. Lo anterior permite observar las variaciones en la estructura de tasas de interés en fechas posteriores al establecimiento de un "corto". Sin embargo, a partir de este análisis no es fácil inferir qué parte de los ajustes en las tasas de interés se deben exclusivamente al cambio en la postura de política monetaria y cuál al ajuste de mercado. En la sección III se describe cómo, utilizando la técnica econométrica de vectores autorregresivos, se puede aislar el componente "activo" de la variable de política monetaria. Adicionalmente, se analiza el impacto de una perturbación restrictiva en la postura monetaria sobre la estructura de tasas de interés, tanto sin aislar la parte "activa" de la política monetaria como aislándola. Finalmente, en la sección IV se presentan algunas conclusiones.

\section{Politica monetaria en México - experiencia reciente.}

En los últimos años, la mayoría de los bancos centrales ha aceptado que la mayor contribución que pueden hacer al desarrollo económico radica en el abatimiento de la inflación 7 . Prueba de ello es el cada vez mayor número de institutos centrales autónomos que tienen el objetivo explícito de procurar la estabilidad de precios. A este respecto, en 1993 se realizaron reformas a la Constitución y otras leyes a fin de otorgar al Banco de México autonomía, con el claro mandato de procurar la estabilidad del poder adquisitivo de la moneda nacional ${ }^{8}$. Dichas reformas buscaban contar con una institución dentro del Estado comprometida con la estabilidad de precios. Esto permite al Banco Central actuar como contrapeso de la administración pública, respecto a situaciones inflacionarias. Adicionalmente, la autonomía también facilita a la autoridad monetaria actuar como contrapeso de los particulares, cuando éstos emprendan acciones que impliquen incrementos de precios o costos.

$\mathrm{Si}$ bien el mandato prioritario de estabilidad de precios es fácilmente cuantificable, las autoridades monetarias no controlan directamente la inflación, ya que ésta es el resultado de un sinnúmero de operaciones y transacciones que se realizan en la economía. Las decisiones de política monetaria tienen un efecto indirecto sobre los distintos componentes que determinan el comportamiento de los precios. En particular, las decisiones de política monetaria modifican tanto las expectativas de los agentes económicos, como las tasas de interés y el tipo de cambio. Esto a su vez incide sobre la demanda agregada, lo cual en su conjunto determina el comportamiento de la inflación (ver Diagrama 19). Lo anterior es la vía por la cual las decisiones de la autoridad monetaria afectan la determinación de los precios y se conoce como mecanismo de transmisión de política monetaria 10 .

\footnotetext{
${ }^{7}$ Ver Pérez López y Schwartz (1999) y Martínez (1998).

8 Banco de México, Ley del Banco de México, Diciembre 1993.

9 Ver O’Dogherty (1997).

10 Para una descripción detallada del mecanismo de transmisión de política monetaria ver Mishkin (1995) y para el caso de México Gil (1997).
} 
Diagrama 1

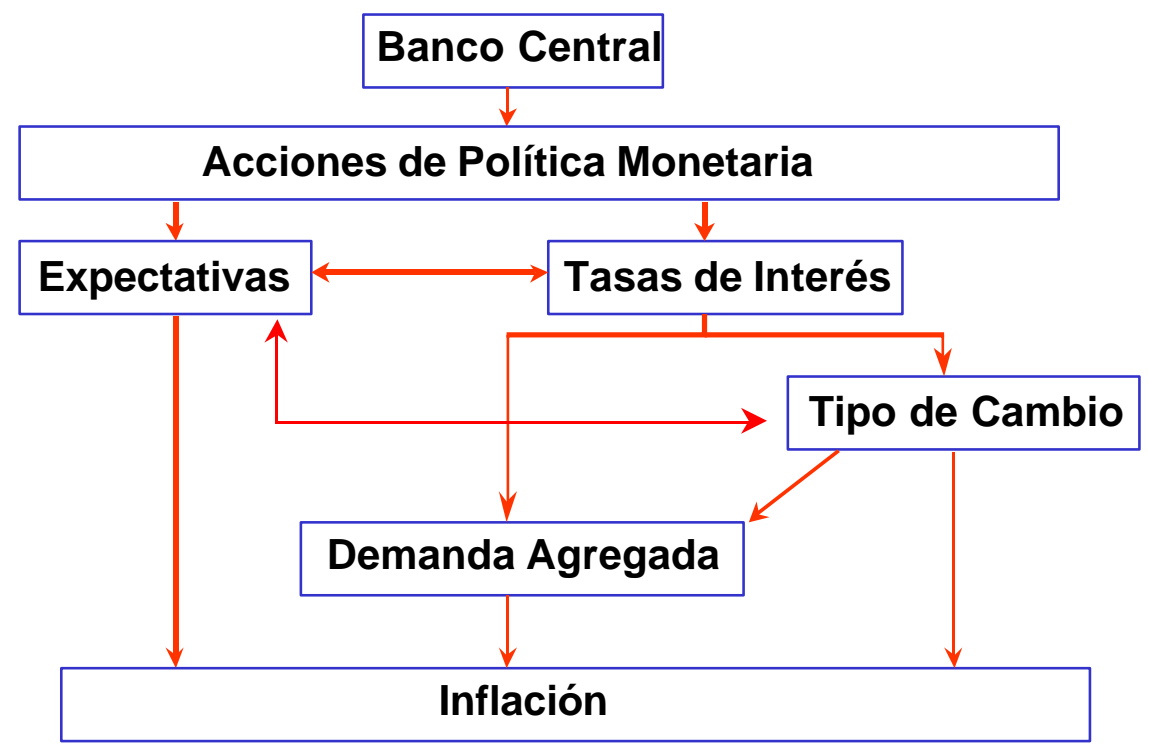

\section{1 Instrumentos de política monetaria.}

Los instrumentos de que dispone un banco central para llevar a cabo su política monetaria son muy diversos, y van desde controles directos al crédito y las tasas de interés, hasta mecanismos indirectos como son los encajes legales y las condiciones bajo las cuales se llevan a cabo las operaciones del banco central en el mercado de dinero (operaciones de mercado abiertol ${ }^{1}$ ). En los últimos años, la mayoría de los bancos centrales ha optado por los instrumentos indirectos de política monetaria, ya que éstos permiten a la autoridad monetaria actuar con mayor eficacia y flexibilidad, especialmente bajo un entorno en el cual las transacciones en los mercados financieros son cada vez mas complejas. En particular, los bancos centrales conducen la política monetaria afectando las condiciones bajo las cuales satisfacen las necesidades de liquidez del mercado de dinero. Estas condiciones dependen tanto de los términos en los que el banco central realiza sus operaciones de mercado abierto (tasas de interés, plazo, tipo de instrumentos, etc.) como de las características del manejo de las cuentas corrientes que mantiene la banca en el banco central (capacidad de sobregiro, tasas de interés de penalización o de remuneración, etc.).

En la mayoría de los países la banca mantiene cuentas corrientes en el banco central, las cuales son utilizadas para hacer frente a sus necesidades de liquidez respecto al mercado de dinero. Esto se debe a que la banca funge como intermediario entre el banco central y el público, a fin de que la demanda de circulante se satisfaga. Por ello, si el público incrementa su demanda por billetes y monedas, el banco central realiza operaciones de mercado abierto a fin de que la banca cuente con la liquidez necesaria en sus cuentas corrientes y pueda retirar el circulante del banco central y colocarlo entre el público. De esta forma, las operaciones de mercado abierto que realizan los bancos centrales buscan

${ }^{11}$ Este tipo de operaciones se refiere a la compra-venta de títulos o subastas de créditos o depósitos que realice el banco central con la intención de modificar la liquidez en el mercado de dinero. 
satisfacer la demanda de base monetaria, la cual corresponde a la suma de billetes y monedas en circulación más los depósitos que mantiene la banca en el banco central. Debido a que los bancos centrales tienen un control directo sobre las operaciones de mercado abierto, a la mayoría de éstos les resulta conveniente utilizar como instrumento de política monetaria, los términos bajo los cuales satisface las necesidades de liquidez en el mercado de dinero.

Para poder evaluar el desempeño de la política monetaria, es fundamental conocer el impacto de las decisiones de la autoridad monetaria sobre los mercados financieros, lo que constituye el primer eslabón en el mecanismo de transmisión de la política monetaria. En este contexto, el Banco de México ha modificado en los últimos años sus instrumentos de política monetaria, buscando no interferir con el libre funcionamiento de los mercados, tanto de dinero como de cambios. La crisis de diciembre de 1994 tuvo dos efectos muy importantes a este respecto: a) forzó la libre flotación del tipo de cambio y b) terminó con una práctica común en el mercado de dinero: la determinación por parte del Banco de México de las tasas de interés a las cuales intervenía diariamente en sus operaciones de mercado abierto. Lo anterior se debió a que los acontecimientos de finales de 1994 y principios de 1995 desataron un marcado incremento en la volatilidad de las tasas de interés, lo que dificultaba la determinación de éstas en las operaciones de mercado abierto. Esta situación dio por resultado un esquema bajo el cual el Banco de México manifiesta su postura de política monetaria a través de señales, por medio de las cuales modifica su participación en el mercado de dinero. Lo anterior ha propiciado que los mercados cambiario y de dinero operen con gran flexibilidad y ello ha permitido mitigar el efecto de perturbaciones externas sobre la actividad productiva.

Las condiciones para el manejo de las cuentas corrientes de la banca en el Banco de México, como lo son la capacidad para incurrir en sobregiros, la posibilidad de contar con un mercado de nivelación posterior al cierre del mercado, entre otras, tienen un impacto directo sobre la demanda de la banca por saldos monetarios en sus cuentas corrientes en el Instituto Central y, por tanto, sobre las condiciones prevalecientes en el mercado de dinero. Lo anterior es importante porque todos los cambios en la demanda y oferta de base monetaria se reflejan en variaciones en los saldos de las cuentas corrientes de la banca en el Banco de México. Así, cuando la demanda de billetes y monedas es mayor que la oferta de dinero primario, esto se traduce en una reducción en el saldo de las cuentas corrientes de la banca en el Banco de México. Esta situación induce incrementos en las tasas de interés, ya que las instituciones de crédito tratarán de cubrir los faltantes en sus cuentas corrientes y evitar así el costo financiero de dichos sobregiros. En el caso contrario, cuando la oferta de dinero primario excede a la demanda, el saldo de las cuentas corrientes de la banca se incrementaría, presionando las tasas de interés a la baja, ya que, al no remunerarse los saldos positivos, las instituciones bancarias tratarán de deshacerse de dichos saldos excedentes.

En cuanto al manejo de las cuentas corrientes de la banca, a partir de marzo de 1995, el Banco de México adoptó el régimen de saldos acumulados, el cual ha sufrido ligeras modificaciones hasta su versión actual. Bajo este régimen, el Instituto Central permite a las instituciones de crédito, al cierre de las 
operaciones del día, registrar sobregiros ${ }^{12}$ en sus cuentas corrientes, siempre y cuando, al término de un periodo de medición de 28 días mantengan un saldo acumulado mayor o igual que cero.

Al final de cada periodo de medición, el Banco de México suma para cada banco los saldos positivos ${ }^{13}$ diarios mantenidos en su cuenta corriente y le resta el total de los sobregiros diarios incurridos, lo cual se conoce como el saldo acumulado de las cuentas corrientes. Si el resultado del cálculo anterior corresponde a un sobregiro neto para una institución de crédito, el Banco de México le cobrará sobre dicho monto dos veces la tasa de interés de CETES a 28 días prevaleciente en el mercado. En el caso contrario, si un banco registra un saldo positivo acumulado en el periodo de medición, éste incurre en un costo indirecto, ya que, al no pagar el Banco de México rendimiento alguno sobre dicho saldo, el banco incurre en el costo de oportunidad de tener esos recursos ociosos. Por ello, conviene a la banca mantener en sus cuentas corrientes un saldo acumulado igual a cero.

Es importante señalar que la mecánica operativa del Banco de México está circunscrita en el contexto del programa monetario, que se publica en enero de cada año. A este respecto, el primer elemento del programa monetario para $2000^{14}$ corresponde a una regla de operación básica orientada a no crear excesos ni faltantes de oferta de base monetaria. De esta manera, el Banco de México se compromete, como norma general, a ajustar diariamente la oferta de dinero primario de forma tal que ésta corresponda en todo momento a su demanda, suministrando los recursos a la tasa que prevalezca en el mercado ${ }^{15}$. En términos técnicos, este criterio equivale a un objetivo de cero en el saldo acumulado de las cuentas corrientes que el Banco de México le lleva a la banca, y corresponde a una postura de política monetaria neutral (ver Diagrama 2).

Adicionalmente, el segundo elemento fundamental del programa monetario de 2000 establece que el Banco de México se reserva el derecho de desviarse de lo establecido en el primer elemento del programa monetario para ajustar su postura de política monetaria. En el caso de que el Instituto Central decidiera restringir su postura de política monetaria, establecería un objetivo de saldos acumulados negativo o "corto". Lo anterior implica que una pequeña porción de la demanda de base monetaria la satisfaga el Banco de México a través de la inducción de un sobregiro de la banca en su cuenta corriente en el Instituto Central (ver Diagrama 2). Es importante señalar que en ningún momento, por vía del "corto", el Banco de México suministra liquidez por un monto inferior al demandado por el mercado de dinero ${ }^{16}$. En este caso, al inducir un sobregiro de la banca en sus cuentas corrientes, el cual de persistir hasta el final del periodo

\footnotetext{
12 Siempre y cuando dichos sobregiros no excedan ciertos límites al cierre del día. Para mayor detalle ver Anexo 4 del Informe Anual del Banco de México, 1996.

${ }^{13}$ Los saldos positivos que pueden compensar sobregiros también están sujetos a ciertos límites diarios.

14 Ver Programa Monetario para 2000, Banco de México.

15 Cualquier desequilibrio en la liquidez que resulte de errores en la estimación diaria de la demanda de base monetaria, es corregido mediante las operaciones que el Banco Central lleva a cabo en el mercado monetario.

16 En el caso de tener que retirar excedentes de liquidez, no lo hace por montos mayores a los demandados por el mercado de dinero.
} 
de medición recibiría una penalización de 2 veces la tasa de interés de CETES a 28 días, repercute en un incremento de las tasas de interés de corto plazo.

\section{Diagrama 2}

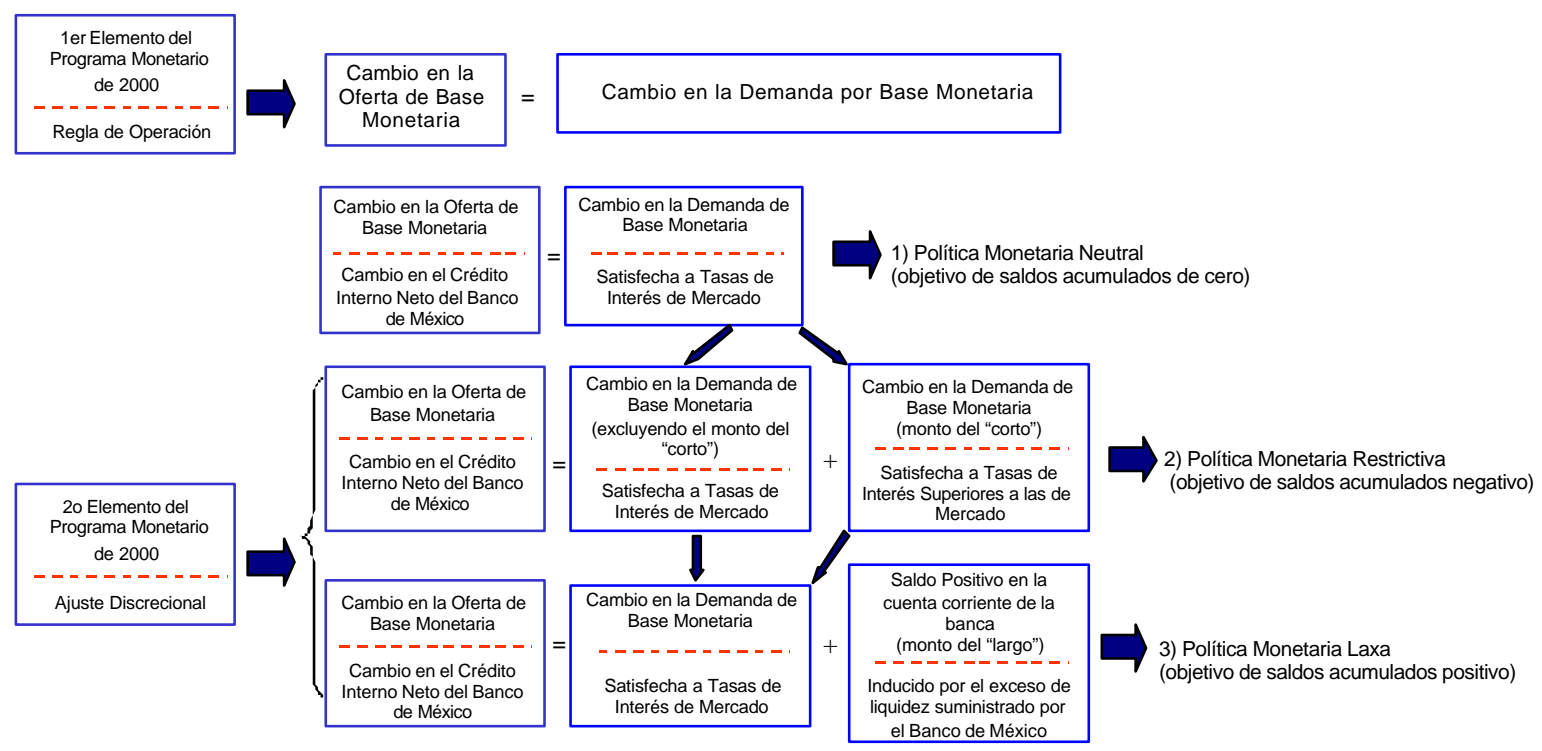

Asimismo, en caso de que el Instituto Central deseara relajar su postura monetaria, mantendría un objetivo de saldos acumulados positivo o "largo". En esta situación, el Banco de México proporcionaría un monto de liquidez mayor que el demandado, lo que se traduciría en saldos positivos en las cuentas corrientes de la banca en el Banco Central (ver Diagrama 2. Así, se inducirían menores tasas de interés de corto plazo al crearse excedentes de liquidez en la banca. No obstante, es importante recordar que en una economía abierta con mercados financieros integrados internacionalmente, como es el caso de México, ningún banco central puede inducir disminuciones permanentes en las tasas de interés por vías distintas del abatimiento de la inflación.

\section{II.2 El uso del objetivo de saldos acumulados como instrumento de politica monetaria.}

Tras la descripción del principal instrumento de política monetaria del Banco de México, el objetivo de saldos acumulados, a continuación se describe el contexto bajo el cual se anunciaron las modificaciones al objetivo de saldos acumulados (i.e."corto") de enero de 1998 a abril de 200017, así como su impacto sobre las tasas de interés. Lo anterior permite analizar la experiencia reciente con el uso del "corto" como instrumento de política monetaria.

Como se podrá apreciar, en la mayoría de los casos, las tasas de interés a todos sus plazos se han incrementado después de la ampliación del "corto", para

$17 \mathrm{El}$ anexo 1 presenta las modificaciones al objetivo de saldos acumulados desde que éste se utiliza como instrumento de política monetaria. 
posteriormente presentar una reducción gradual. No obstante lo anterior, han sido muy variadas las situaciones bajo las cuales se ha hecho uso del "corto", así como la magnitud de su efecto sobre las tasas de interés. Sin embargo, con base en este análisis no se puede inferir qué parte de las variaciones en las tasas de interés se debe a las modificaciones en la postura de política monetaria y cuál al ajuste del mercado. Por ello, en la sección III, se pretende estimar qué parte de los ajustes a la política monetaria se debe a la reacción del Instituto Emisor ante acontecimientos previamente descontados por los mercados (efecto "reacción"), y cuál a un componente que se podría identificar como uno de política monetaria "activa".

Cabe destacar que la descripción que se presenta a continuación sobre las condiciones bajo las cuales se modificó la postura de política monetaria no pretende hacer ningún tipo de evaluación sobre la conveniencia de su adopción. Se limita a describir el entorno bajo el cual se aplicaron dichas modificaciones, así como el comportamiento de las tasas de interés en fechas anteriores y posteriores a la variación del "corto".

\section{II.2.1 Restricciones en la postura de politica monetaria, "cortos", durante 199818 .}

En los primeros meses de 1998, el tipo de cambio se depreció como resultado de la crisis asiática y de la caída en los precios del petróleo. Lo anterior causó un deterioro de las expectativas inflacionarias, las cuales adicionalmente resintieron el incremento en los precios de la tortilla y de la masa en febrero de dicho año. En respuesta a lo anterior, el 11 de marzo el Banco de México restringió su postura de política monetaria pasando de un objetivo de saldos acumulado de 0 a -20 millones de pesos (m.p.). Con una política monetaria restrictiva, el Banco de México busca incrementar las tasas de interés, lo cual se debe reflejar en la estructura de tasas de interés de los títulos gubernamentales colocados en el mercado primario (CETES) ${ }^{19}$.

La Gráfica 1 presenta la estructura de tasas de interés de los valores gubernamentales (CETES) ${ }^{20}$ de las dos semanas previas al anuncio de la ampliación del "corto" (líneas 1 y 2), así como de las siguientes tres subastas primarias (líneas 3, 4 y 5). Como se puede apreciar, tras el anuncio de la restricción monetaria se incrementaron las tasas de interés a todos sus plazos. No obstante, para la segunda y tercera semanas posteriores al "corto", sólo las tasas de corto plazo se mantenían en niveles superiores a los registrados con anterioridad al anuncio de la restricción monetaria.

\footnotetext{
18 Ver Informe Anual, Banco de México, 1998.

19 Es importante destacar que el presente ejercicio utiliza los resultados de las subastas primarias de valores gubernamentales, las cuales se realizan los martes de cada semana. Por ello, es de esperarse que el impacto de una ampliación del "corto" sobre las tasas de interés pueda apreciarse con mayor claridad en el mercado secundario. No obstante, no se cuenta con una serie histórica de la curva de rendimiento de los valores gubernamentales en el mercado secundario.

${ }^{20}$ La estructura de tasas de interés se construye estimando una tasa de rendimiento para las fechas que se encuentran entre dos plazos de vencimiento de la subasta primaria. Lo anterior se logra a través de la tasa implícita o equivalente para los plazos intermedios.
} 


\section{Gráfica 1}

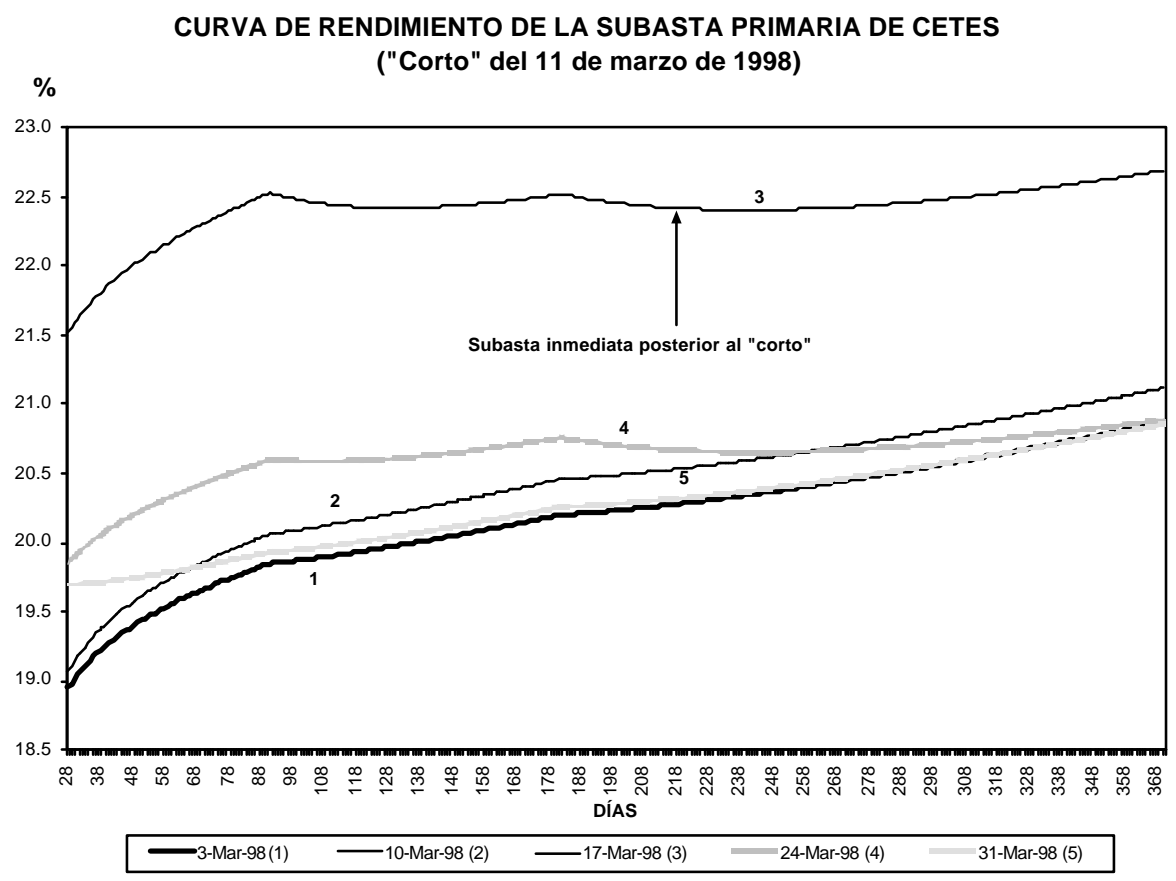

Durante la segunda quincena de mayo y junio, el entorno internacional continuó empeorando, lo que presionó el mercado cambiario y, en consecuencia, las expectativas de inflación, las cuales se deterioraron aún más ante un incremento adicional en los precios de la tortilla y la masa. El Banco de México reaccionó ampliando el "corto" de -20 a -30 m.p. el 25 de junio.

\section{Gráfica 2}

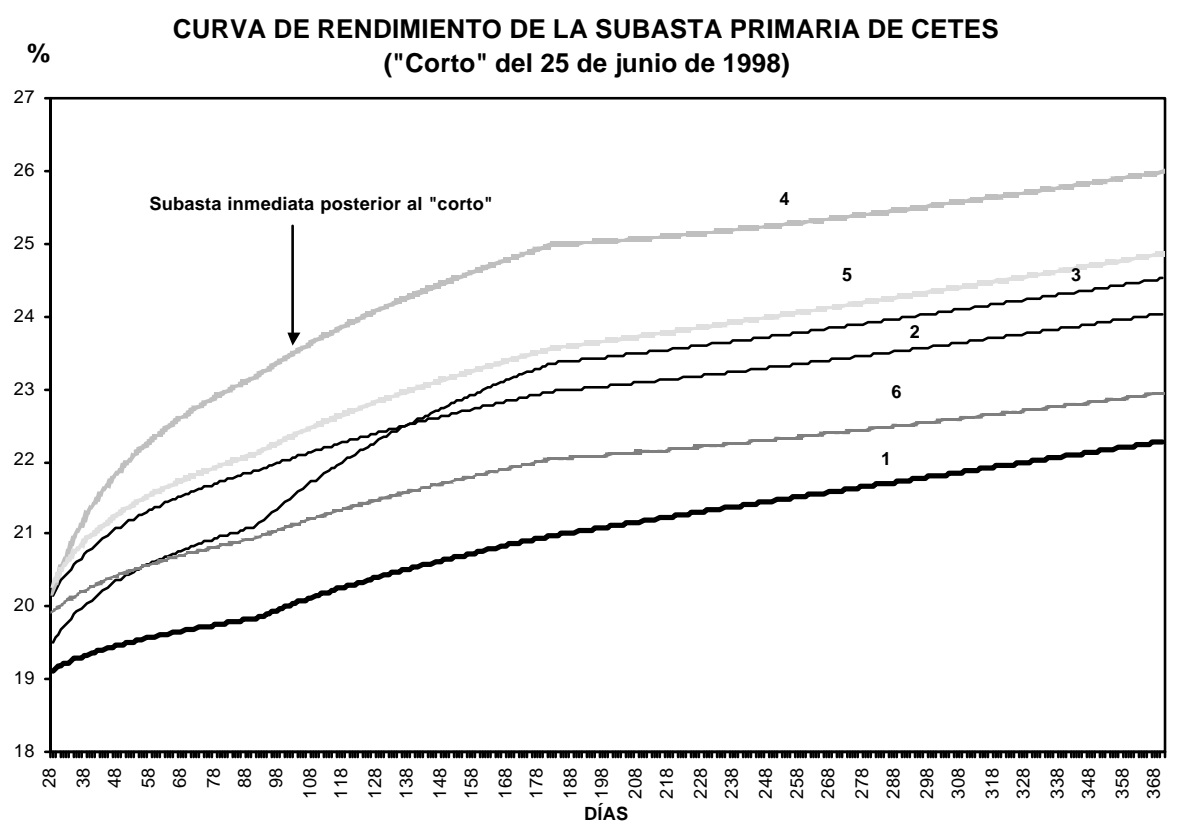

-9-Jun-1998 (1) —-16-Jun-1998 (2) $\quad$-23-Jun-1998 (3) $\quad-$ 30-Jun-1998 (4) $\quad$ 7-Jul-1998 (5) $\quad$ - 14-Jul-1998 (6) 
Como se puede apreciar en la Gráfica 2, las tasas de interés de los CETES en todos sus plazos se incrementaron significativamente dos semanas antes del anuncio de la restricción monetaria. Esto refleja el incremento en la percepción de riesgo, descontada por el mercado, en los instrumentos de deuda denominados en moneda nacional (la curva de rendimiento pasó de la línea 1 a la 2). No obstante, en la semana previa a la ampliación del "corto" las tasas de interés de corto plazo ( 28 y 91 días) mostraron contracciones importantes. Tras el anuncio de la modificación del "corto" las tasas de interés se incrementaron en todos sus plazos (línea 4), para posteriormente disminuir en las siguientes semanas.

En agosto, la moratoria rusa sobre su deuda interna y externa modificó sensiblemente la percepción de riesgo de las economías emergentes, lo cual se tradujo en fuertes presiones sobre la cotización de la moneda nacional. En respuesta a lo anterior, el Banco de México reaccionó restringiendo progresivamente su postura de política monetaria. La restricción del 10 de agosto (el "corto" pasó de -30 a -50 m.p.) no tuvo más que un ligero impacto sobre las tasas de interés, tal como se puede apreciar en la Gráfica 3 (cambio en la curva de rendimiento de la línea 1 a la 2). Por ello, el 17 de agosto el Banco de México reaccionó ampliando aún más el "corto", pasando éste de -50 a -70 m.p. A pesar de lo anterior, la mayor restricción monetaria no indujo incrementos en las tasas de interés (la curva de rendimiento de la línea 2 a la 3 quedó prácticamente inalterada).

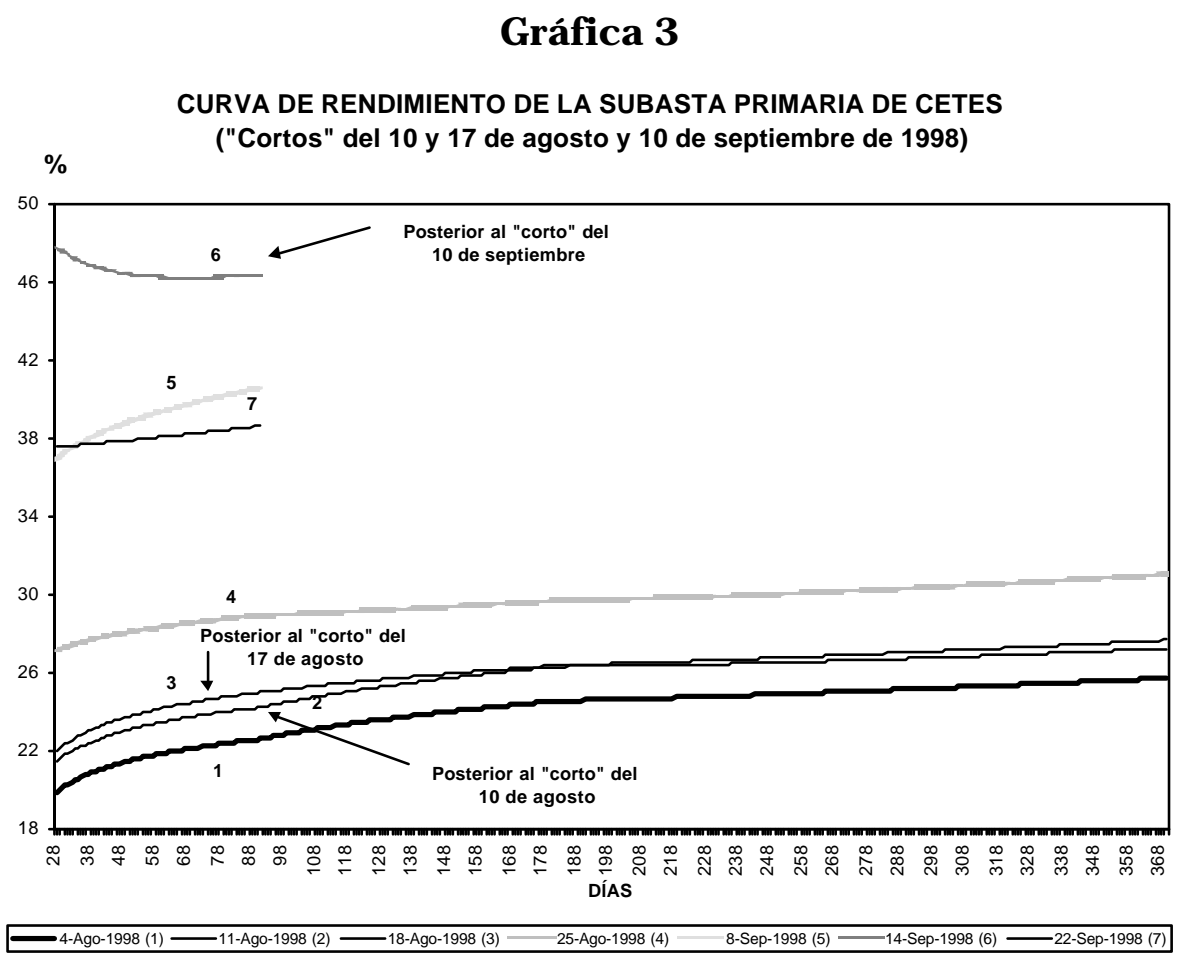

Es oportuno explicar porqué la ampliación del "corto" dejó de inducir incrementos en las tasas de interés. La modificación del objetivo de saldos acumulados por 
parte del Banco de México es una señal de que el Instituto Central desea observar incrementos en las tasas de interés, especialmente en las de corto plazo. No obstante, es la banca la que finalmente determina tanto la magnitud como la permanencia de las variaciones en las tasas de interés.

Adicionalmente, se ha observado que el impacto del "corto" en las tasas de interés está influenciado por la posición de liquidez que mantenga el Banco de México respecto a la banca en el mercado de dinero. Cuando el Instituto Central mantiene una posición líquida frente al mercado de dinero, se convierte en el proveedor marginal de recursos a través de sus operaciones de mercado abierto. Lo anterior se conoce como posición acreedora del Banco de México respecto de la banca. En el caso contrario, el Banco de México es un deudor del mercado de dinero y tiene que retirar los excesos de liquidez vía sus operaciones diarias. Cuando el Banco Central mantiene una posición acreedora frente al mercado de dinero le resulta más fácil inducir incrementos en las tasas de interés, ya que es el proveedor único de la liquidez que requiere la banca para saldar sus cuentas corrientes sin incurrir en sobregiro alguno. De manera análoga, cuando el Banco de México mantiene una posición deudora frente al mercado de dinero, es la banca la que tiene mayor control sobre la determinación de las tasas de interés, ya que ésta mantiene la liquidez necesaria para contener incrementos en dichas $\operatorname{tasas}^{21}$.

Durante 1998, la posición del Banco de México respecto al mercado de dinero pasó de ser fundamentalmente acreedora a deudora, lo que debilitó la efectividad del "corto" para ejercer presiones al alza en las tasas de interés. Lo anterior se hizo patente tras el anuncio de las ampliaciones del "corto" del 10 y 17 de agosto. A este respecto, la Gráfica 4 muestra la posición diaria del Banco de México respecto al mercado de dinero. Como se puede apreciar, durante agosto de 1998 la posición del Banco de México fue mayoritariamente deudora (las cifras negativas representan retiros de liquidez en las operaciones de mercado abierto), lo que posiblemente impidió que el "corto" funcionara adecuadamente para inducir incrementos en las tasas de interés. Ante la situación antes descrita, el Banco Central redujo la capacidad de sobregiro intradía de los bancos con el Banco de México en el Sistema de Pagos Electrónicos de Uso Ampliado (SPEUA) e impuso un piso a las tasas de interés para sus operaciones de mercado abierto los tres días hábiles posteriores al 21 de agosto22. $\mathrm{Si}$ bien estas medidas propiciaron incrementos en las tasas de interés, sólo se adoptaron de manera transitoria. Por ello, el Banco de México se vio en la necesidad de buscar otras alternativas para incrementar la efectividad del "corto".

\footnotetext{
${ }^{21}$ Lo anterior se puede complicar más cuando la banca puede ejercer crédito intradía (ofrecido por el Banco de México a través del Sistema de Pagos Electrónicos de Uso Ampliado - SPEUA), ya que algunos intermediarios pueden utilizar dichos recursos para evitar incrementos en las tasas de "fondeo" o de muy corto plazo.

22 Fue posible imponer pisos a las tasas de interés debido a que la Tesorería de la Federación recauda impuestos en dichos días, lo que implica un depósito en la cuenta del gobierno en el Banco de México con recursos que se mantenían previamente en la banca. Esta extracción temporal de liquidez ofrece durante unos días una posición acreedora al Instituto Central.
} 


\section{Gráfica 4}

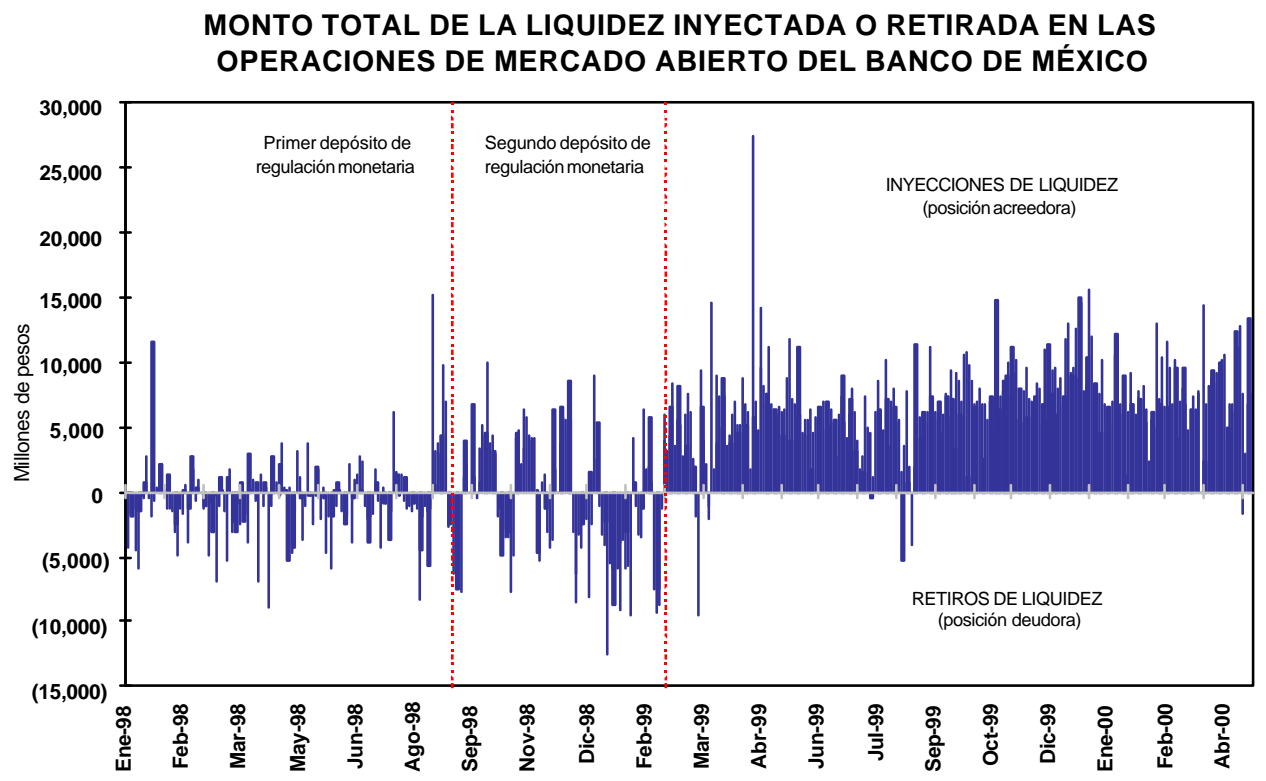

A este respecto, el Banco de México decidió reforzar sus instrumentos de política monetaria y contar con una posición acreedora diaria respecto al mercado de dinero. Para ello, estableció que a partir del 2 de septiembre las instituciones de crédito estaban obligadas a constituir un depósito en el Banco de México a plazo indefinido, por 1,250 m.p. diarios hasta llegar a un total de 25,000 m.p. ${ }^{23}$, remunerado a la tasa de interés interbancaria de equilibrio (TIIE) a 28 días. Para evitar que el referido depósito implicara un sobregiro de las instituciones de crédito con el Banco de México, el Instituto Central anunció que repondría toda la liquidez que se llegase a retirar por esta vía mediante operaciones de mercado abierto a muy corto plazo. Esto permitía al Banco Central contar con una posición acreedora diaria respecto al mercado de dinero, ya que si bien la operación no tenía impacto monetario, el Instituto Central podía financiar su posición deudora a largo plazo y contar con un sobrante de liquidez que le regresaría al mercado a muy corto plazo. Lo anterior permitió reforzar la efectividad del "corto" para inducir incrementos en las tasas de interés.

El posterior deterioro de las condiciones externas dio por resultado un incremento en las tasas de interés. De hecho, la primera subasta primaria de septiembre fue declarada desierta ante el incremento solicitado en las tasas de interés. En los primeros días de septiembre se agudizó aún más la percepción de riesgo sobre las economías emergentes. En particular sobre la economía brasileña y su posible contagio a la economía mexicana, lo que presionó aún más el mercado cambiario ${ }^{24}$. Ante lo anterior, el 10 de septiembre la Comisión de Cambios $^{25}$ decretó una venta discrecional de 278 millones de dólares²6, en adición

${ }^{23}$ La distribución del depósito obligatorio entre las distintas instituciones de crédito se realizaría a prorrata en función de sus pasivos totales al cierre de junio.

24 Las presiones sobre el mercado cambiario se agudizaron debido a que algunos inversionistas cubrían sus posiciones de riesgo en Brasil a través de posiciones cortas en pesos mexicanos.

25 Compuesta por funcionarios de la Secretaría de Hacienda y Crédito Público y del Banco de México.

26 Banco de México, Boletín de Prensa 98 de 1998. 
a los 200 millones de dólares que se subastan de manera automática cuando el tipo de cambio se deprecia durante el día en más de 2 por ciento27. La intervención anterior fue complementada con la ampliación del "corto" de -70 a -100 m.p. Como se puede observar en la Gráfica 3, en esta ocasión la mayor restricción monetaria, aunada a las medidas que fortalecieron la efectividad del "corto", dieron por resultado un sensible incremento en las tasas de interés (la curva de rendimiento pasó de la línea 5 a la (38). Lo anterior, junto con una situación internacional más favorable contribuyó a revertir la depreciación del tipo de cambio ${ }^{29}$ y a estabilizar las expectativas inflacionarias, lo que permitió la gradual reducción de las tasas de interés (ver Gráfica 3).

No obstante, ante el impacto inflacionario de la turbulencia financiera, el 30 de noviembre el Banco de México decidió intensificar nuevamente la restricción monetaria, pasando el "corto" de -100 a -130 m.p. Esto se dio para frenar la inercia de las expectativas de inflación y para hacer frente al impacto inflacionario de los incrementos en los precios públicos (realizados para contrarrestar los menores ingresos por la exportación de crudo). La restricción monetaria buscaba aumentar las posibilidades de alcanzar la meta de inflación de 13 por ciento para 1999. Por ello, se puede decir que esta ampliación del "corto" es cualitativamente distinta a las anteriores.

\section{Gráfica 5}

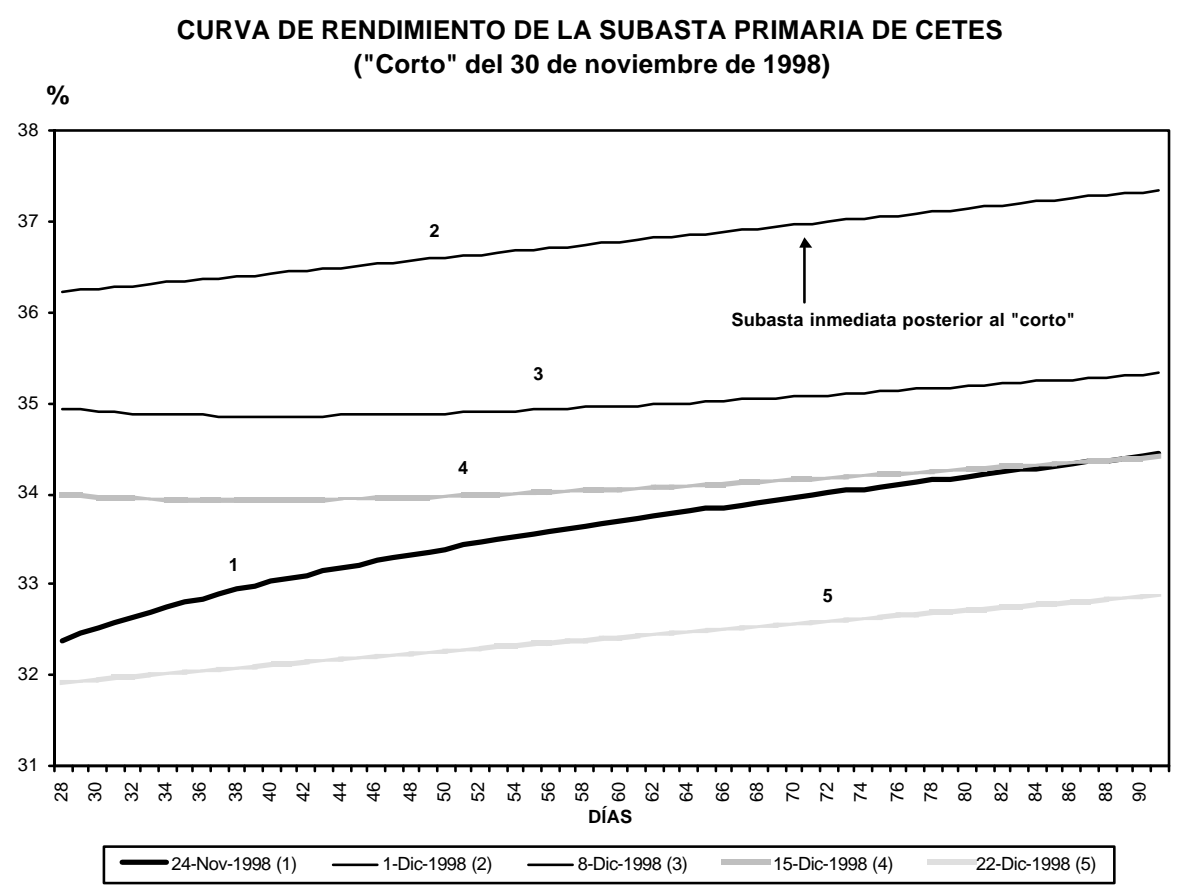

Como se muestra en la Gráfica 5, tras la restricción monetaria del 30 de noviembre, las tasas de interés registraron un incremento significativo en todos

27 Informe Anual, Banco de México, 1997, pag. 130-132.

${ }^{28}$ Las curvas de rendimiento que sólo llegan a 91 días se deben a la ausencia de emisión de CETES a 182 y 364 días.

29 Los incrementos en las tasas de interés de corto plazo encarecieron las posiciones cortas en moneda nacional, lo que redujo la especulación sobre la posible depreciación del tipo de cambio. 
sus plazos. Es importante destacar que tres semanas después del anuncio de la ampliación del "corto", las tasas de interés, en todos sus plazos, se encontraban en niveles inferiores a los registrados antes de dicho anuncio. Esta decisión de política monetaria, aunada al mejoramiento del entorno internacional, ilustra cómo el objetivo de saldos acumulados puede, a través de la inducción de incrementos temporales en las tasas de interés, reducir las expectativas de inflación ${ }^{30}$, permitiendo con ello la reducción gradual de las tasas de interés.

\section{II.2.2 Restricciones en la postura de politica monetaria, "cortos", durante 199931 .}

A finales de 1998 y principios de 1999, el problema cambiario en Brasil representaba el mayor factor de riesgo para las economías emergentes, en especial para América Latina. Esta situación dio por resultado la depreciación del real el 13 de enero de 1999, y la posterior flotación de dicha moneda, lo cual repercutió significativamente en la cotización del peso mexicano. En respuesta a lo anterior, el Banco de México decidió restringir su postura de política monetaria, ampliando el "corto" de -130 a -160 m.p., el 13 de enero de 1999. Esta medida buscaba, a través de propiciar incrementos en las tasas de interés, frenar la depreciación del peso y conservar la posibilidad de tener una inflación de 13 por ciento al cierre del año.

Se puede observar en la Gráfica 6 que la curva de rendimiento tenía una pendiente negativa, como resultado de la marcada incertidumbre de corto plazo asociada con el régimen cambiario en Brasil. Adicionalmente, se puede apreciar que, con anticipación al anuncio de la ampliación del "corto", las tasas de interés de corto plazo se habían incrementado (la curva de rendimiento pasó de la línea 1 a la 2. Es decir, la determinación de las tasas de interés sin la intervención directa del Banco de México había dado por resultado un ajuste en las mismas, el cual incorporaba la incertidumbre asociada con la modificación del régimen cambiario en Brasil. Tras el anuncio del "corto" y bajo un entorno eminentemente volátil, las tasas de interés registraron incrementos aún mayores. En las semanas posteriores a la ampliación del "corto", se redujo paulatinamente la incertidumbre en los mercados internacionales y nacionales, lo que permitió la apreciación del tipo de cambio y la reducción gradual de las tasas de interés, tanto nominales como reales ${ }^{32}$.

\footnotetext{
30 Las expectativas de inflación para los siguientes doce meses publicadas por Infosel, pasaron de $17.1 \%$ el 27 de noviembre a $16.4 \%$ el 18 de diciembre.

31 Ver Informe Anual, Banco de México, 1999.

32 En febrero de 1999, se adoptó un segundo depósito obligatorio de la banca en el Banco de México. En adición a lo anterior, el Banco de México decidió colocar valores gubernamentales a largo plazo (Bondes) a través de sus operaciones de mercado abierto. Con el depósito obligatorio y la venta de títulos de largo plazo, el Instituto Central retira liquidez del mercado de dinero a largo plazo, y la compensa con operaciones de mercado abierto de corto plazo (por ejemplo, subastas de crédito a un día). Esto permite al Banco de México mantener una posición acreedora diaria respecto al mercado de dinero.
} 


\section{Gráfica 6}

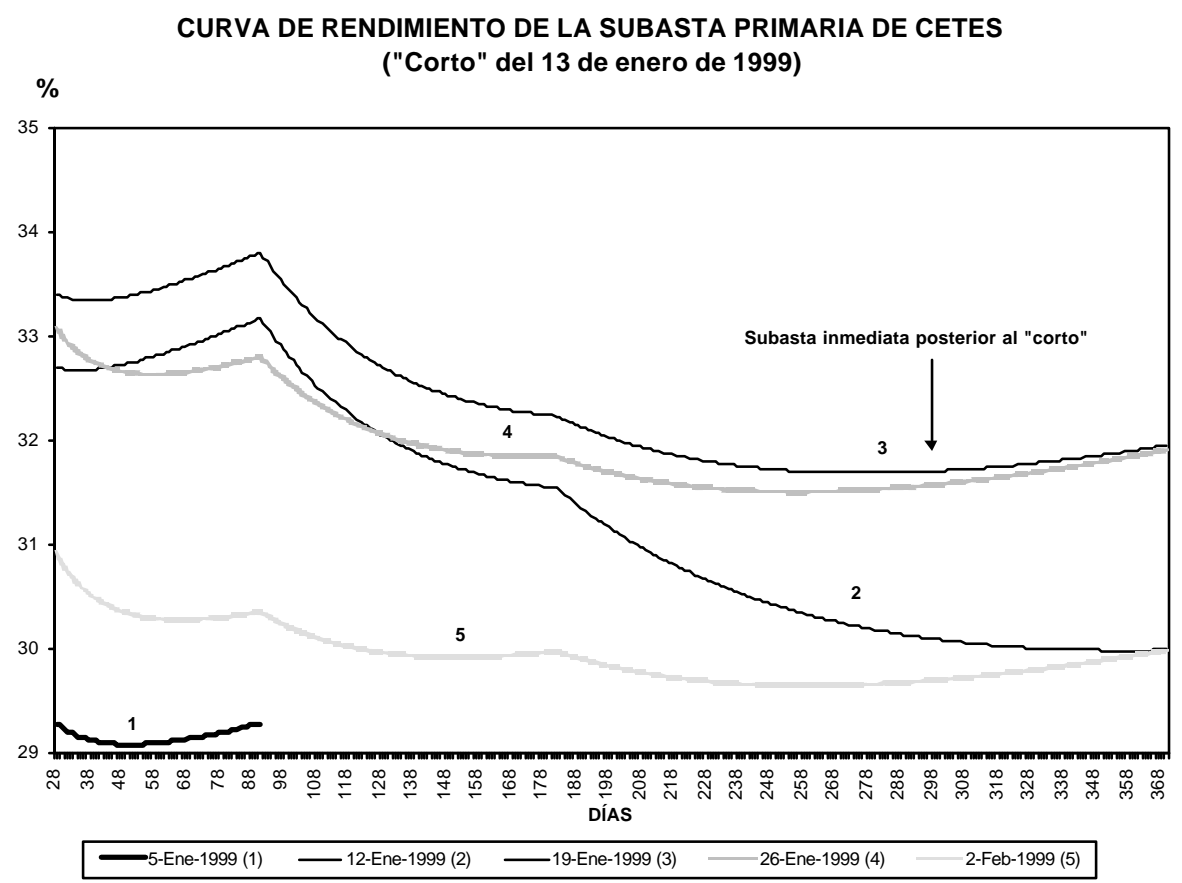

\section{II.2.3 Restricciones en la postura de politica monetaria, "cortos", durante 2000 (enero-abril)33.}

Para el año 2000, el Banco de México planteó como meta una inflación que no excediera de 10 por ciento. Adicionalmente, anunció su intención de lograr que en 2003 la inflación nacional converja con la de los principales socios comerciales del país. Considerando que durante las primeras semanas del año se presentaron acontecimientos que podrían haberse traducido en presiones inflacionarias incompatibles con las metas anteriores, el Instituto Central decidió restringir su postura de política monetaria, pasando de un "corto" de -160 a uno de -180 m.p., el 18 de enero. Los principales factores por los cuales se decidió restringir la política monetaria fueron: a) el carácter no recurrente de diversos factores que permitieron el descenso de la inflación en 1999 (por ejemplo, la apreciación del tipo de cambio y la reducción de los precios de algunos productos agropecuarios); b) la permanencia de expectativas de inflación superiores a la meta oficial; esto último es de especial importancia si se considera que a principios del año se lleva a cabo una fracción importante de las negociaciones salariales contractuales; y c) los incrementos en los primeros días del año de diversos precios, cuyo comportamiento resultaba incompatible con la meta de inflación.

33 Ver Informe sobre la inflación, Banco de México, enero - marzo 2000. 
Como se puede apreciar en la Gráfica 7, las tasas de interés de la subasta coincidente con la modificación del "corto" reaccionaron con un ligero incremento. No obstante, para la siguiente semana el aumento fue aún mayor. En las tres subastas posteriores, las tasas de interés se redujeron paulatinamente hasta llegar a niveles inferiores al de la subasta previa al anuncio del "corto" 34 .

\section{Gráfica 7}

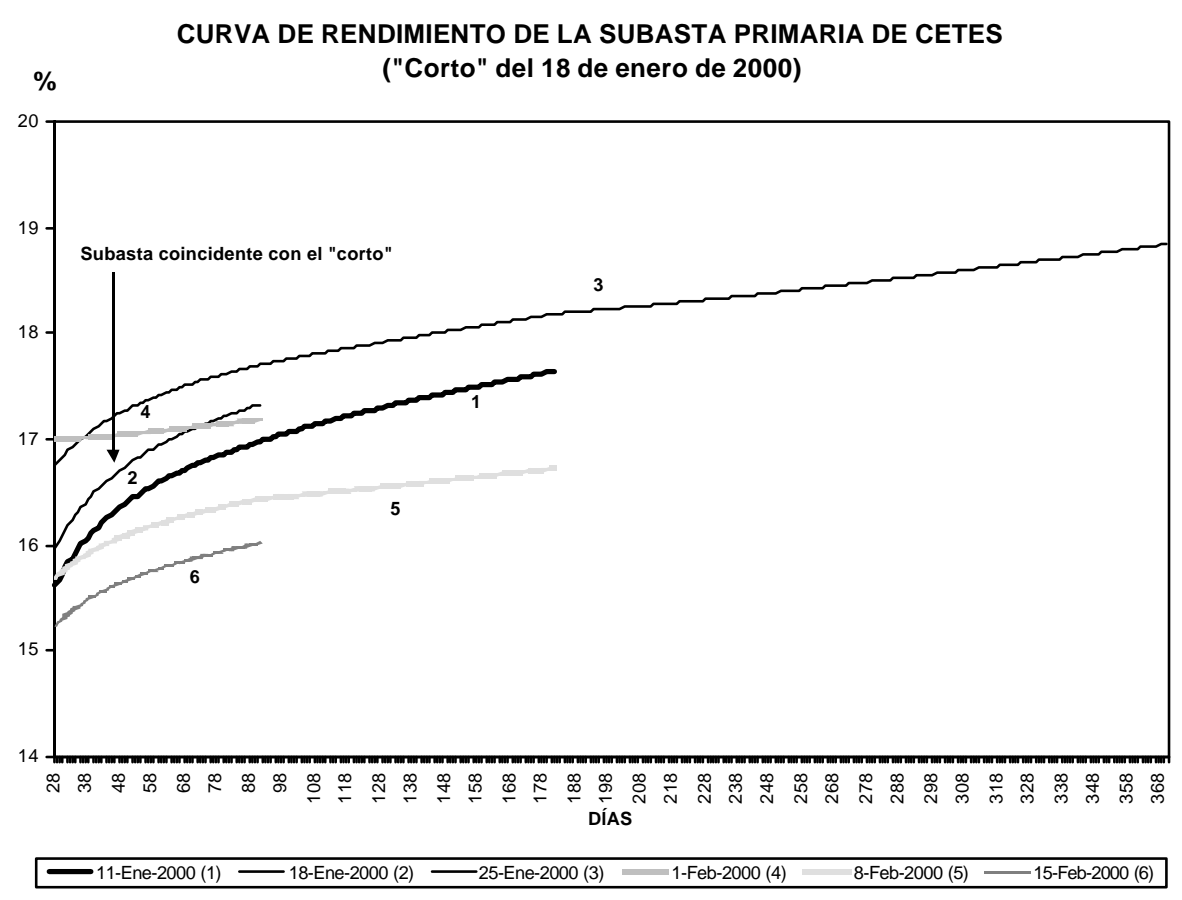

Durante 1998, 1999 y los primeros meses de 2000, se observa que en la mayoría de los casos, tanto las tasas de interés de corto como de largo plazo se elevaron en fechas posteriores a la modificación de la postura de política monetaria y que dichas tasas disminuyeron gradualmente unas cuantas semanas después. A este respecto, es importante señalar que las variaciones en las tasas de interés en todos sus plazos reflejan, entre otras cosas, cambios en las primas de riesgo y de liquidez. Este último concepto es especialmente importante para los instrumentos a mayores plazos ya que éstos tienden a ser poco líquidos, en especial en situaciones de incertidumbre y cuando se presentan incrementos generalizados en las tasas de interés.

Como se vio en esta sección, las modificaciones de la postura de política monetaria en los últimos años han ocurrido bajo condiciones internas y externas muy distintas. Adicionalmente, si se considera que el régimen cambiario de libre flotación y la libre determinación de tasas de interés permite al mercado ajustarse

34 Cabe destacar que para 2000 la colocación de CETES a 6 meses y un año se realiza con menor frecuencia que en años anteriores, aunque con emisiones mayores. 
a las condiciones prevalecientes en la economía, no es fácil inferir qué parte de los ajustes en tasas de interés responde exclusivamente al cambio de postura de política monetaria y cuál al ajuste del mercado. Por ello, en la siguiente sección se utiliza un herramental econométrico que permite aislar el componente "activo" de la política monetaria, con el fin de poder determinar el impacto de dicho componente sobre la estructura de tasas de interés.

\section{Efecto de la politica monetaria sobre las tasas de interés.}

Como se ha mencionado en secciones anteriores, el anuncio del objetivo de saldos acumulados tiene como finalidad mandar una señal sobre la postura de política monetaria del Banco de México. Un objetivo de saldos acumulados negativo o "corto" se traduce en una señal de incremento en las tasas de interés para el mercado de dinero, un objetivo de saldos acumulados igual a cero implica neutralidad ante las tasas de interés $\mathrm{y}$, finalmente, un objetivo de saldos acumulado positivo o "largo" implica una señal que induciría una caída en las tasas de interés.

De acuerdo a los lineamientos seguidos por el Banco de México en los últimos años ${ }^{35}$, la postura de política monetaria puede ser modificada hacia una tendencia más restrictiva ante las siguientes circunstancias: a) cuando se detecten presiones inflacionarias futuras que sean incongruentes con los objetivos de inflación; b) cuando se presenten perturbaciones inflacionarias exógenas; c) cuando se necesite restaurar condiciones ordenadas en los mercados cambiario y de dinero y d) cuando las expectativas de inflación se desvíen considerablemente respecto al objetivo. Es importante mencionar que al materializarse algunos de estos eventos, los mercados inducirían por sí solos aumentos en las tasas de interés, los cuales se presentarían en adición al efecto que sobre dichas tasas tenga un ajuste en la postura de política monetaria. Por el contrario, si dichos eventos no han sido descontados suficientemente por el mercado ni dado lugar a un ajuste en las tasas de interés a satisfacción del Banco Central, sería de esperarse que tras el anuncio de una restricción monetaria se presentaran incrementos en las tasas de interés.

De esta forma, se puede establecer que un cambio en la postura de política monetaria puede deberse a: i) una respuesta o "reacción" del Banco Central ante eventos internos o externos fácilmente observables por el público y con posibles impactos inflacionarios, los cuales es de esperarse que por sí mismos induzcan incrementos en las tasas de interés. A lo anterior lo llamaremos efecto "reacción", y ii) a una decisión por parte del Instituto Central de modificar su postura de política monetaria, en respuesta a presiones inflacionarias que no hayan sido previamente descontadas por el mercado a satisfacción del Instituto Central. A este último caso le llamaremos politica monetaria "preventiva" o "activa".

Para descomponer movimientos en el "corto" en su efecto "reacción" y su componente "activo" se utilizó la metodología propuesta por ChristianoEichenbaum-Evans ${ }^{36}$. Estos autores descomponen a la variable de política

35 Ver Programa Monetario para 2000, Banco de México.

36 Ver Christiano, Eichenbaum y Evans (1994a, 1994b) y Eichenbaum y Evans (1992). 
monetaria estadounidense (tasa de interés de fondos federales) en su componente de "reacción" y su componente "activo". Dicha metodología se basa en la técnica econométrica de vectores autorregresivos (VAR) y, en particular, en las llamadas funciones impulso-respuesta ${ }^{37}$.

Es importante mencionar que varios autores han analizado el efecto de la política monetaria, medido a través de cambios en el objetivo de saldos acumulados, sobre las tasas de interés de corto plazo para el caso de México. A este respecto Juan-Ramón (1996), Aguilar y Juan-Ramón (1997) y Greenham (1997) utilizan la técnica econométrica de mínimos cuadrados ordinarios para evaluar el efecto de la política monetaria sobre las tasas de interés de corto plazo. Adicionalmente, Greenham (1997) hace uso de vectores autorregresivos en su análisis, lo cual permite capturar posibles interrelaciones dinámicas entre las variables involucradas.

El presente trabajo difiere de los anteriores estudios en dos aspectos fundamentales: 1) evalúa el impacto de la política monetaria, sobre todo la estructura de tasas de interés y 2) presenta una descomposición explícita del componente "activo" y de "reacción" de la variable de política monetaria. Lo anterior permite evaluar si la política monetaria en México en los últimos años ha tenido un componente "activo" y estudiar el impacto de una política monetaria exógena sobre la estructura de tasas de interés.

\section{1 Descomposición de la variable de política monetaria en sus componentes de "reacción y "activo".}

Con la finalidad de facilitar la exposición del análisis empírico primero se describen las variables que se utilizaron en la estimación de los VARs. Dichas variables se pueden dividir en tres categorías: 1) variables que buscan capturar el efecto "reacción"; 2) variables de política monetaria y 3) variables que caracterizan la estructura de tasas de interés. Las variables de "reacción" se incluyen para capturar las condiciones prevalecientes en los mercados. Se supone que para el caso de los mercados mexicanos, las variables que pueden capturar el efecto "reacción" son: a) el tipo de cambio spot venta (TC); b) el rendimiento del Bono Global 2026, como variable que refleja el riego país (BG2026); c) las expectativas de inflación publicadas por Infosel para los siguientes 12 meses $^{38}$ (EXPINF) y d) la tasa de interés del Bono del Tesoro de E.U.A. a 30 años (TB30).

Como variable de política monetaria se utiliza el objetivo de saldos acumulados (CORTO). Como se vio anteriormente, dicha variable refleja la postura de política monetaria del Banco de México. Finalmente, la tercera categoría la integran las

\footnotetext{
37 La técnica de VAR consiste en la estimación de un sistema de ecuaciones en donde cada variable está explicada por rezagos de sí misma y rezagos del resto de las variables. De esta manera, todas las variables del sistema son consideradas potencialmente endógenas. Por su parte, las funciones impulso-respuesta, que se obtienen a partir de la estimación del VAR, corresponden a la respuesta de cada variable ante perturbaciones no esperadas en alguna de las variables incluidas en el VAR. Para una explicación detallada de vectores autorregresivos ver Christiano, Eichenbaum y Evans (1997) y Leeper, Sims y Zha (1996).

38 Obtenidas a través de una encuesta semanal que se publica los viernes, realizada a 20 analistas del mercado de dinero.
} 
variables que caracterizan la estructura de tasas de interés, compuesta por las tasas de interés de los CETES del mercado primario a 28 (C28), 91 (C91), 182 (C182) y 364 (C364) días.

Como ya se mencionó, el objetivo es analizar el impacto de una ampliación en el "corto" (política monetaria restrictiva) sobre la estructura de tasas de interés, descontando el efecto "reacción" sobre dichas tasas. Para lo anterior, se utilizaron las funciones impulso-respuesta de las tasas de interés a sus distintos plazos ante una perturbación en la variable de política monetaria (CORTO). Es importante señalar que la identificación de dichas funciones requiere establecer un orden para las variables incluidas en los VARs, el cual en este caso está asociado con el grado de exogeneidad de las variables entre si39. Para efectos del presente estudio se requiere que las variables de "reacción" se coloquen antes del "corto" y las variables que representan a la estructura de tasas de interés se ubiquen después de dicha variable de política monetaria 40 .

De esta forma, el orden entre las variables del VAR utilizado es: TB30-BG2026-TC-EXPINF-CORTO-CETESi, donde $i=28,91,182$ y 364 . Lo anterior se fundamenta en el hecho de que la variable de política monetaria (CORTO) por un lado responde contemporáneamente a movimientos en el tipo de cambio, en las expectativas de inflación, en el riesgo país y en las tasas de interés externas y por otro, tiene efectos en el mismo periodo sobre la estructura de tasas de interés.

Para analizar la respuesta de la estructura de tasas de interés ante una perturbación en el componente "activo" de la variable de política monetaria (CORTO) es necesario aislar dicho componente "activo". Para este fin, el primer paso es suponer que la variable de política monetaria está determinada por:

$$
\operatorname{CORTO}_{t}=\varphi\left(\theta_{t}\right)+u_{t}
$$

Donde $\varphi_{t}$ es una función lineal, $\theta_{t}$ es el conjunto de datos disponibles para la autoridad monetaria al determinar $C O R T O_{t}$ y $u_{t}$ es el error estructural o perturbación de política monetaria "activa" (i.e. exógeno), no correlacionado con el conjunto de información $\theta_{t}$.

Como se demostrará más adelante, utilizando la técnica de vectores autorregresivos y, en particular a las funciones impulso-respuesta, la variable de política monetaria se puede descomponer en los siguientes dos efectos:

$$
\text { CORTO }{ }_{t}=\text { efecto reacción }+ \text { efecto activo }
$$

donde: efecto reacción $=\varphi\left(\theta_{t}\right)$

$$
\text { efecto activo }=u_{t}
$$

\footnotetext{
39 Para mayor detalle sobre la identificación de las funciones impulso-respuesta ver Hamilton, capítulo 11 .

$40 \mathrm{El}$ orden que entre sí mantengan las variables de "reacción" no altera los resultados de las funciones impulso-repuesta de las tasas de interés ante una perturbación en el "corto". Ver Bernanke y Blinder (1992).
} 
A continuación se describe formalmente el vector autorregresivo y el uso de las funciones impulso-respuesta para aislar el componente "activo" del "corto".

Se denomina $Z_{t}$ al vector (6x1) que incluye a las variables de cada VAR en el tiempo $t$, en el orden establecido. De esta forma:

$$
Z_{t}^{\prime}=\left(T B 30_{t}, B G 2026_{t}, \text { TC }_{t}, \text { EXPINF }_{t}, \text { CORTO }_{t}, \text { CETESi }_{t}\right)
$$

donde $i=28,91,182$ y 364

Se supone a continuación el siguiente modelo estructural de primer orden:

$$
A_{0} Z_{t}=a+A_{1} Z_{t-1}+U_{t}
$$

donde $a$ es un vector de constantes (6x1), $A_{i}$ son matrices de coeficientes (6x6) y $U_{t}$ es el vector de errores estructurales (6x1). Se supone que los errores estructurales no presentan correlación serial $\left(E\left(U_{t} U_{s}\right)=0\right.$ cuando $\left.t \neq s\right)$ y tampoco correlación contemporánea $\left(E\left(U_{t} U_{t}\right)=\Sigma\right.$, siendo $\Sigma$ una matriz diagonal). A esto último se le conoce como ortogonalidad en los errores estructurales.

Es importante mencionar que el modelo estructural de la ecuación (4) no puede ser estimado econométricamente debido a que las variables incluidas tienen efectos contemporáneos entre sí11. Por lo anterior, es necesario transformar el modelo estructural a su versión reducida:

$$
Z_{t}=b+B Z_{t-1}+V_{t}
$$

donde $b=A_{0}^{-1} a, B=A_{0}^{-1} A_{1}$ y $V_{t}=A_{0}^{-1} U_{t}$.

De esta forma, los errores del modelo reducido, $V_{t}$, son una combinación lineal de los errores estructurales.

$$
V_{t}=C U_{t}
$$

donde $C=A_{0}^{-1}$

Adicionalmente, se supone que $\mathrm{C}$ es una matriz triangular inferior y con la unidad en la diagonal, que los errores del modelo reducido, $V_{t}$, no tienen correlación serial y tienen una matriz de varianza-covarianza $\Omega=C \Sigma C^{\prime}$. Debido a que $C$ no es una matriz diagonal sino triangular inferior, $\Omega$ no es una matriz diagonal y, por tanto, permite correlación contemporánea entre los errores $V_{t}$. Es decir, no hay ortogonalidad entre los errores del modelo reducido.

Los supuestos sobre la matriz C son necesarios para la identificación de las funciones impulso-respuesta $y$ están fundamentados por criterios de

${ }^{41}$ Para una explicación detallada sobre este tema ver Enders (1995) pag. 294-295. 
exogeneidad $^{42}$. La estructura de la matriz $\mathrm{C}$ implica que el elemento $\mathrm{j}$ del vector $V_{t}$ está correlacionado con los primeros $\mathrm{j}$ elementos del vector $U_{t}$ pero es ortogonal al resto de los elementos de dicho vector. Con la finalidad de clarificar el punto anterior a continuación se desarrolla explícitamente la ecuación (6).

donde, $i=28,91,182$ y 364

$$
\left(\begin{array}{l}
v_{\text {TB30 }} \\
v_{B G 2026} \\
v_{T C} \\
v_{\text {EXPINF }} \\
v_{\text {CORTO }} \\
v_{\text {CETESi }}
\end{array}\right)=\left(\begin{array}{cccccc}
1 & 0 & 0 & 0 & 0 & 0 \\
C_{21} & 1 & 0 & 0 & 0 & 0 \\
C_{31} C_{32} & 1 & 0 & 0 & 0 \\
C_{41} C_{42} & C_{43} & 1 & 0 & 0 \\
C_{51} C_{52} & C_{53} & C_{54} & 1 & 0 \\
C_{61} C_{62} & C_{63} & C_{64} & C_{65} & 1
\end{array}\right) *\left(\begin{array}{l}
u_{T B 30} \\
u_{B G 2026} \\
u_{T C} \\
u_{\text {EXPINF }} \\
u_{\text {CORTO }} \\
u_{\text {CETESi }}
\end{array}\right)
$$

De esta forma,

$$
v_{\text {CORTO }}=C_{51} u_{T B 30}+C_{52} u_{B G 2026}+C_{53} u_{T C}+C_{54} u_{\text {EXPINF }}+u_{\text {CORTO }}
$$

Por tanto, $v_{\text {CоRто }}$ (el error del "corto" en el modelo reducido) está correlacionado con $u_{T B 30}, u_{B G 2026}, u_{T C}, u_{\text {EXPINF }}$ y $u_{\text {CORTO }}$ pero es ortogonal a $u_{C E T E S i}$. Es de esta forma cómo la parte no esperada de la variable de política monetaria estimada por el VAR, $v_{\text {СоRто }}$, se puede desagregar en dos componentes, el efecto "reacción" $\left(C_{51} u_{T B 30}+C_{52} u_{B G 2026}+C_{53} u_{T C}+C_{54} u_{E X P I N F}\right)$ y el efecto de política monetaria "activa" $\left(u_{\text {СОRTO }}\right)$, los cuales están determinados por los errores que provienen del modelo estructural.

Para ilustrar la descomposición de la variable de política monetaria en su efecto "reacción" y su efecto "activo", a continuación se presenta la quinta ecuación del sistema reducido (5), donde el error de dicha ecuación, $v_{\text {Совто }}$, se expresa utilizando la ecuación (7).

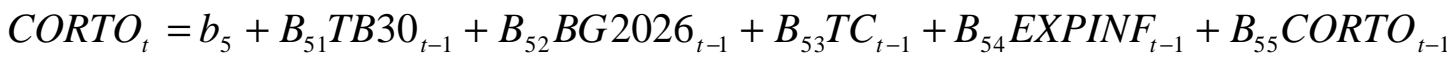

$$
\begin{aligned}
& B_{56} \text { CETESi }_{t-1}+C_{51} u_{T B 30}+C_{52} u_{B G 2026}+C_{53} u_{T C}+C_{54} u_{\text {EXPINF }}+u_{\text {CORTO }}
\end{aligned}
$$

donde $i=28,91,182$ y 364

Recordando la ecuación (1):

$$
\operatorname{CORTO}_{t}=\varphi\left(\theta_{t}\right)+u_{t}
$$

42 Ver Hamilton capítulo 11. 
y utilizando la ecuación (8) podemos establecer que:

$$
\begin{gathered}
\varphi\left(\theta_{t}\right)=b_{5}+B_{51} \text { TB30 }_{t-1}+B_{52} \text { BG2026 } \\
B_{56} \text { CETESi }_{t-1}+C_{51} u_{\text {TB } 30}+C_{52} u_{\text {BG } 2026}+C_{53} u_{T C}+B_{54} \text { EXPINF }_{t-1}+B_{55} u_{\text {EXPINF }} \\
u_{t}=u_{\text {CORTO }}
\end{gathered}
$$

De esta forma, $\varphi\left(\theta_{t}\right)$ corresponde al componente de política monetaria de "reacción", esperado y no esperado. Esto se debe a que incluye el efecto esperado,

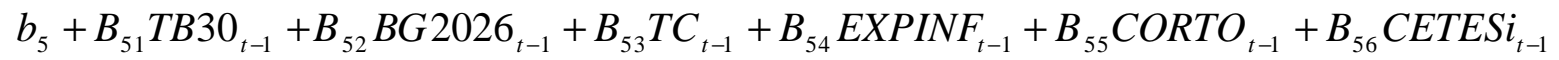
y el no esperado, $C_{51} u_{T B 30}+C_{52} u_{B G 2026}+C_{53} u_{T C}+C_{54} u_{\text {EXPINF}}$, de las variables de "reacción" sobre el "corto". Por su parte, $u_{\text {СоRто }}$ representa al componente "activo" o exógeno de la política monetaria, que tampoco es esperado.

Una vez estimados los VARs anteriores, se pueden obtener las funciones impulsorespuesta que permiten analizar el efecto de perturbaciones en el "corto" sobre la estructura de tasas de interés. Es importante mencionar que las funciones impulso-respuesta se obtienen a través de perturbaciones no esperados en los errores estructurales $\left(U_{t}\right)$, es decir, a través de perturbaciones en los errores ortogonales, en este caso $u_{\text {СОRTO }}{ }^{43}$. De esta forma, al estimar las funciones impulso-respuesta antes mencionadas se estaría analizando la respuesta de la estructura de tasas de interés, ante una perturbación en el componente "activo" de política monetaria.

Es importante mencionar que si no se incluyen las variables de "reacción" dentro de cada VAR, el efecto atribuible a una perturbación en el "corto" incluirá tanto a la parte "activa" como a la de "reacción". En otras palabras, al estimar un VAR omitiendo las variables de "reacción", el error de la variable de política monetaria no se puede descomponer en su parte de "reacción" y en su parte "activa". Lo anterior debido a que por construcción se supone que el impacto de una ampliación del "corto" sobre las tasas de interés es directo y no está relacionado con otras variables 44 .

\section{III.2 Análisis Empírico.}

Con la finalidad de analizar el impacto de la política monetaria sobre la estructura de tasas de interés, se estimaron dos grupos de VARs, uno sin incluir

43 Lo señalado obedece al hecho de que si los errores están correlacionados contemporáneamente, como es el caso de los errores del modelo en su forma reducida, $V_{t}$, no es posible aislar el efecto de una perturbación en la variable j sobre la variable i (ver Hamilton pag 318-323).

44 En este caso, la variable de política monetaria estaría determinada por: CORTO $_{t}=b_{1}+B_{11}$ CORTO $_{t-1}+B_{12}$ CETESi $_{t-1}+\eta_{\text {СRTO }}$, donde $i=28,91,182$ y 364 . Es importante enfatizar el hecho de que $\eta_{\text {совто }}$ representa la parte no explicada por esta ecuación y que puede estar determinada por movimientos en variables como el tipo de cambio y las expectativas de inflación, entre otras. De lo anterior se desprende el hecho de que en la estimación de esta ecuación, el término $\eta_{\text {совто }}$ incluye tanto a la parte de "reacción" de la política monetaria (no incluida explícitamente a través de variables explicativas en el modelo) como a la parte "activa". 
las variables de "reacción" y otro incorporándolas. Lo anterior con el fin de ilustrar la importancia de incluir al efecto "reacción" en la respuesta de la estructura de tasas de interés ante un cambio en la variable de política monetaria. Para efectos del presente análisis y con los fundamentos detallados en la sección III.1; el orden de las variables incluidas en los VARs es el siguiente45: 1) CORTO-CETESi, para el grupo que no incluye el efecto "reacción" y 2) TB30-BG2026-TC-EXPINF-CORTO-CETESi, para el que sí lo incluye, para ambos casos $i=28,91,182$ y 364 . El periodo de estudio comprende del 11 noviembre de 199746 al 25 de abril de 2000 y la periodicidad de los datos es semanal 47 .

A partir de la estimación de los VARs anteriores se pueden obtener las funciones impulso-respuesta de la curva de rendimiento de tasas de interés ante una perturbación no esperada en la variable de política monetaria (CORTO). Para el primer grupo de VARs, que no considera las variables de "reacción", la perturbación no esperada en el "corto" incluye tanto al componente de política monetaria "activa" como al del efecto "reacción". Por su parte, para el segundo grupo, la perturbación de política monetaria corresponde únicamente al componente de política monetaria "activa" puesto que ya se "controló" por los componentes de "reacción". A continuación se presentan los resultados de las funciones impulso-respuesta derivadas de la estimación de los VARs antes mencionados 48 .

\section{IV.1 Resultado de los VARs sin incluir las variables de "reacción".}

La Gráfica 8 muestra las funciones impulso-respuesta de las tasas de interés a sus diferentes plazos ante una ampliación no esperada en el "corto". Lo anterior corresponde a una ampliación en el "corto" de aproximadamente 5.5 millones de pesos 49 . Dichas funciones se obtuvieron a partir de la estimación del primer

${ }^{45}$ La definición de las variables se presenta en la sección III.1.

${ }^{46}$ La muestra inicia a partir de que se empezaron a publicar las expectativas de inflación semanales de Infosel.

47 Para las tasas de interés de los CETES del mercado primario, tipo de cambio, "corto", tasa de interés del Bono Global 2026 y tasa de interés del Bono del Tesoro de E.U.A. se utilizó el dato del martes de cada semana, correspondiente al día de la subasta primaria de papel gubernamental. Para el caso de las expectativas de inflación se utilizó el dato del viernes anterior al día de la subasta primaria. Finalmente, en el caso de que hubiera un cambio en el "corto" y éste fuera en lunes o martes, se registró el martes de esa semana y si el cambio en el "corto" se dio de miércoles a viernes, se registró el siguiente martes. Para las subastas primarias de CETES del 8 de septiembre de 1998 al 5 de enero de 1999, período cuando no hubo emisión de los CETES a 182 y 364 días, se utilizó la tasa de interés de los CETES a 91 días. Por su parte, a partir del 6 de julio de 1999 las emisiones de CETES a 182 y 364 días se realizan quincenalmente y a partir del 28 de marzo de 2000 las emisiones a 364 días tienen una periodicidad mensual. Por tanto, a fin de mantener la periodicidad semanal para estos plazos se repitió el dato quincenal para las dos semanas correspondientes y el mensual para las semanas del mes.

48 En la estimación de los VARs, un rezago fue suficiente para que los errores no presentaran correlación serial. Dicha estimación se realizó en niveles ya que todas las variables son integradas de orden uno (se incluyó constante y tendencia en la prueba de Dickey-Fuller Aumentada para aquellos casos en donde resultaron significativas ). Se encontró evidencia de cointegración entre las series incluidas en cada VAR. Estas pruebas se presentan en el anexo 2.

49 La magnitud exacta de una perturbación de una desviación estándar en el "corto" depende de cada VAR. Para el VAR que incluye a la tasa de interés de CETES a 28 días es de 5.56 m.p. y para los VARs que incluyen a las tasas de interés de CETES a 91, 182 y 364 es de 5.53 m.p. 
grupo de VARs y se graficaron para un periodo de 20 semanas. De esta manera, la Gráfica 8.1 se obtuvo de la estimación que incluye a la tasa de interés de CETES a 28 días, la Gráfica 8.2 de la que incorpora a la tasa de interés de CETES a 91 días, la Gráfica 8.3 de la que considera a la tasa de interés de CETES a 182 días y, finalmente la Gráfica 8.4 proviene de la estimación del VAR con la tasa de interés de CETES a 364 días. Las líneas punteadas indican la banda de un intervalo de confianza al 95\%.

\section{Gráfica 8}
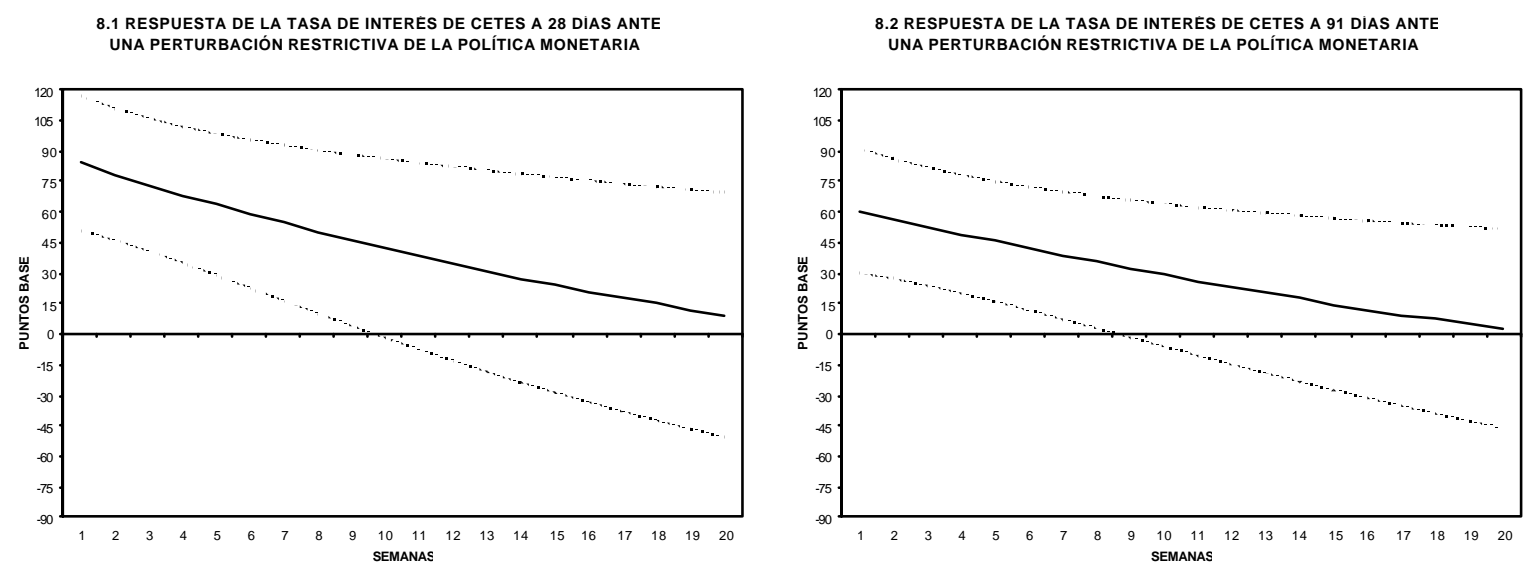

8.3 RESPUESTA DE LA TASA DE INTERES DE CETES A 182 DIAS ANTE UNA PERTURBACIÓN RESTRICTIVA DE LA POLITICA MONETARIA
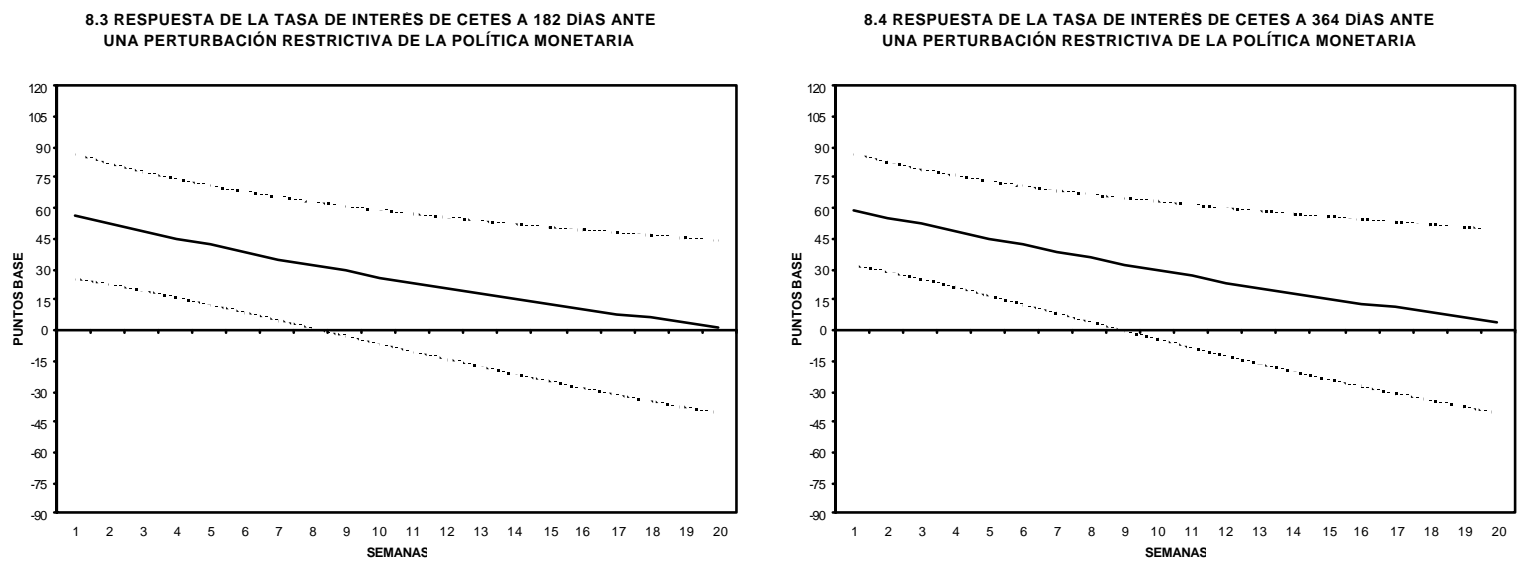

Como es de esperarse, una perturbación restrictiva en la política monetaria, tiene un efecto inicial positivo y significativamente diferente de cero en la estructura de tasas de interés, el cual va disminuyendo con el paso del tiempo. Por ejemplo, el incremento en la tasa de interés de los CETES a 28 días es estadísticamente significativo durante las primeras nueve semanas después de la ampliación del "corto" (el intervalo al 95\% de confianza no incluye al cero) y su magnitud va disminuyendo gradualmente. Para el resto de las tasas de interés se aprecia que a partir de la octava semana, dicho efecto se vuelve estadísticamente no significativo (ver Gráficas 8.2, 8.3 y 8.4).

Es importante destacar que el impacto de una perturbación restrictiva en la política monetaria es menor conforme aumenta el plazo de las tasas de interés. De esta forma, el impacto de una ampliación del "corto" es mayor sobre la tasa de interés de CETES a 28 días (Gráfica 8.1) que sobre la de 364 días (Gráfica 8.4), 
siendo el efecto inicial sobre este último plazo menor en aproximadamente 25 puntos base respecto al de 28 días.

La respuesta de las tasas de interés a diferentes plazos se puede apreciar con mayor claridad al graficar el efecto de una política monetaria restrictiva sobre toda la curva de rendimiento50. Con la finalidad de presentar una perturbación homogénea para todas las tasas de interés y similar a las restricciones monetarias anunciadas recientemente, se decidió analizar una perturbación en el "corto" de 20 m.p. en vez de utilizar el correspondiente a una desviación estándar51. Lo anterior se puede observar en la Gráfica 9.

Para poder simular el impacto sobre la curva de rendimiento de una política monetaria restrictiva, es necesario partir de una curva de rendimiento inicial (línea 1). En este caso, se decidió que dicha curva estuviera representada por el promedio de las tasas de interés para sus diferentes plazos durante el período del 25 de enero al 25 de abril del 200052. De esta forma, en la Gráfica 9.1, el primer punto de la línea 1 corresponde al promedio de la tasa de interés de CETES a 28 días durante el periodo antes señalado, el segundo punto al promedio de la tasa de interés de CETES a 91 días durante el mismo periodo y así para el resto de los plazos.

\section{Gráfica 9}
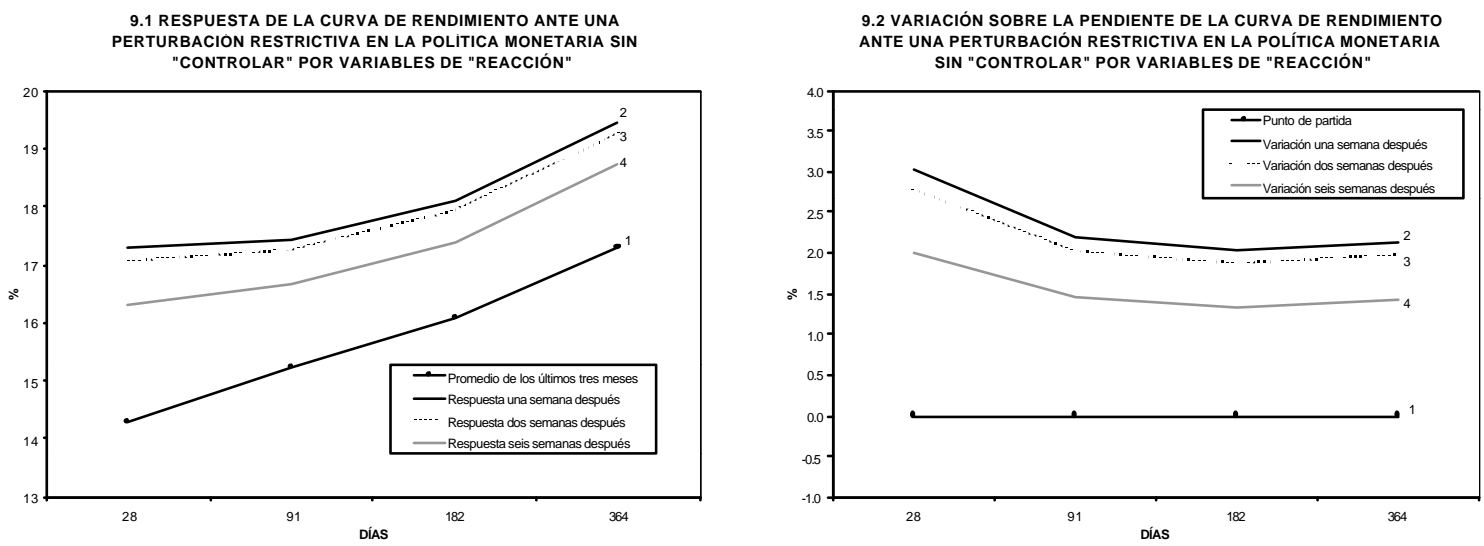

La línea 2 es la respuesta de la curva de rendimiento una semana después de la ampliación del "corto" en 20 m.p. Este resultado se obtiene de agregar a la curva de rendimiento inicial la respuesta de las tasas de interés a cada uno de sus plazos tras una semana de la perturbación de política monetaria restrictiva, estimada a partir de las funciones impulso-respuesta ilustradas en las Gráficas 8.1, 8.2, 8.3 y 8.4, pero con una perturbación en el "corto" de 20 m.p. Por ejemplo, el promedio para la tasa de interés de CETES a 28 días durante el período de tres meses antes señalado fue de $14.28 \%$ y la respuesta de dicha tasa

50 Edelberg y Marshall (1996) realizan un estudio similar para Estados Unidos.

51 La respuesta de las diferentes tasas de interés se estimó proporcionalmente a partir de las funciones impulso-respuesta para una perturbación no esperada en el "corto" de aproximadamente 5.5 m.p.

$52 \mathrm{El}$ mismo análisis se puede realizar tomando otra curva de rendimiento inicial, como puede ser la curva de rendimiento de la última subasta primaria. 
de interés una semana después de la ampliación del "corto" en 20 m.p. fue de 3.01 puntos porcentuales. Por tanto, una semana después de la referida perturbación monetaria restrictiva, la tasa de interés de CETES a 28 días resulta de $17.3 \%$. La misma metodología se aplicó para el resto de los plazos.

Las líneas 3 y 4 corresponden a la respuesta de la curva de rendimiento ante una política monetaria restrictiva de 20 m.p., después de dos y seis semanas, respectivamente. Como se puede apreciar en la Gráfica 9.1, después de 6 semanas el impacto de una ampliación en el "corto" continúa siendo importante. Adicionalmente, se puede observar que una política monetaria restrictiva tiene un efecto positivo sobre la curva de rendimiento a todos sus plazos, el cual va disminuyendo conforme pasa el tiempo y es mayor sobre las tasas de interés a menor plazo.

Para poder apreciar con claridad el efecto de una ampliación en el "corto" sobre la pendiente de la curva de rendimiento se presenta la Gráfica 9.2, la cual muestra el incremento en las tasas de interés a sus diferentes plazos ante la restricción monetaria. En dicha Gráfica se iguala a cero la curva de rendimiento original (línea 1). Las líneas 2, 3 y 4 de la Gráfica 9.2 representan la variación de la curva de rendimiento una, dos y seis semanas después de la ampliación del "corto" en 20 m.p., respectivamente. Así, el primer punto de la línea 2 de la Gráfica 9.2 corresponde a la variación en la tasa de interés de CETES a 28 días tras la ampliación del "corto" en 20 m.p. (3.01 puntos porcentuales). En la Gráfica 9.2 destaca el incremento en las tasas de interés de corto plazo por encima del correspondiente a plazos mayores.

A la luz de estos resultados se puede decir que el "corto" tiene: a) un impacto positivo y estadísticamente significativo en las tasas de interés a sus diferentes plazos y b) un mayor impacto sobre las tasas de interés de corto plazo. Sin embargo, al no haber "controlado" por las variables de "reacción" en la estimación de los VARs, no es posible diferenciar qué parte de los movimientos en la variable de política monetaria se debe a una reacción de las autoridades monetarias ante el entorno prevaleciente en los mercados, y cual a una decisión de política monetaria "activa", no descontada por el mercado a satisfacción del Banco de México. Por ello, de los resultados anteriores no se puede determinar qué proporción de la respuesta de las tasas de interés a diferentes plazos se debe al componente de política monetaria "activa", y cual a las condiciones prevalecientes en los mercados (efecto "reacción"). En la siguiente sección se presenta la estimación del segundo grupo de VARs. Dichos VARs incluyen a las variables de "reacción" y, por tanto, pueden aislar la respuesta de las tasas de interés ante un cambio en la política monetaria "activa".

\section{IV.2 Resultado de los VARs incluyendo las variables de "reacción".}

La Gráfica 10 presenta las funciones impulso-respuesta para las tasas de interés a sus diferentes plazos ante una perturbación no esperada, de una desviación estandar, en el "corto", controlando por las variables de "reacción". Dicha 
desviación estándar es de aproximadamente 4.9 m.p53. Las funciones impulsorespuesta se obtuvieron a partir de la estimación del segundo grupo de VARs, para un periodo de 20 semanas. Como en la sección anterior, la Gráfica 10.1 se obtuvo de la estimación que incorpora a la tasa de interés de CETES a 28 días, la Gráfica 10.2 de la que incluye a la tasa de interés de CETES a 91 días y así para el resto de las gráficas. Es importante recordar que en este caso la perturbación en el "corto" corresponde al componente de política monetaria "activa". Las líneas punteadas indican la banda de un intervalo al 95\% de confianza.

Como se puede apreciar en la Gráfica 10, aún "controlando" por las variables del efecto "reacción", una perturbación no esperada en el "corto", continúa teniendo un efecto inicial positivo y estadísticamente significativo sobre las tasas de interés a sus diferentes plazos. Al igual que en el ejercicio anterior, el efecto es mayor sobre las tasas de interés de menor plazo y disminuye con el paso del tiempo.

\section{Gráfica 10}

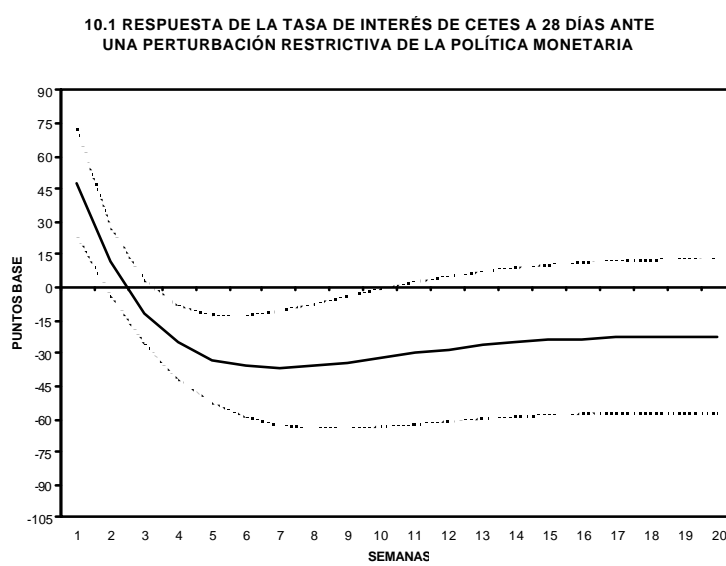

10.2 RESPUESTA DE LA TASA DE INTERES DE CETES A 91 DIAS ANTE UNA PERTURBACIÓN RESTRICTIVA DE LA POLITICA MONETARIA

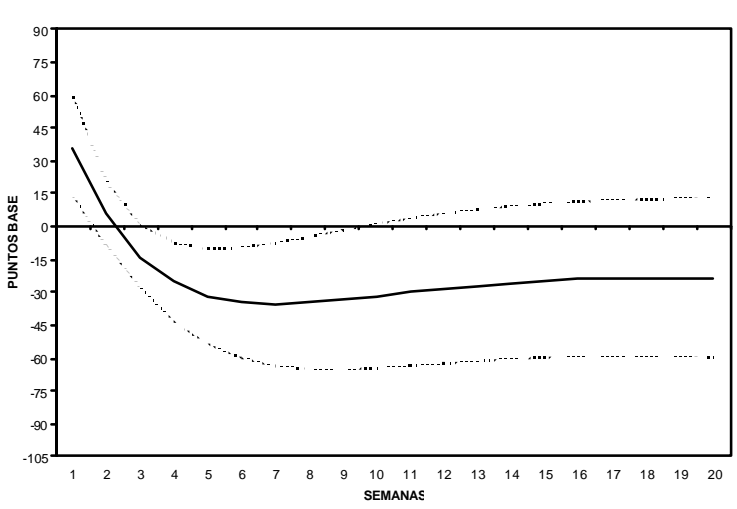

10.3 RESPUESTA DE LA TASA DE INTERES DE CETES A 182 DIAS ANTE UNA PERTURBACIÓN RESTRICTIVA DE LA POLITICA MONETARIA

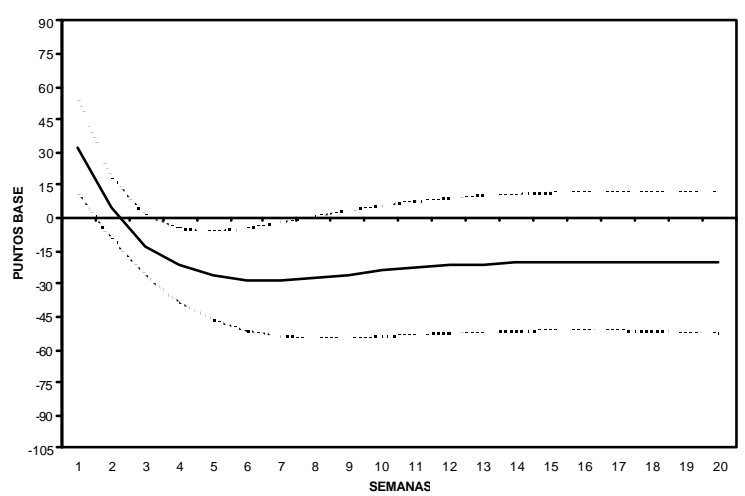

10.4 RESPUESTA DE LA TASA DE INTERES DE CETES A 365 DIAS ANTE UNA PERTURBACIÓN RESTRICTIVA DE LA POLITICA MONETARIA

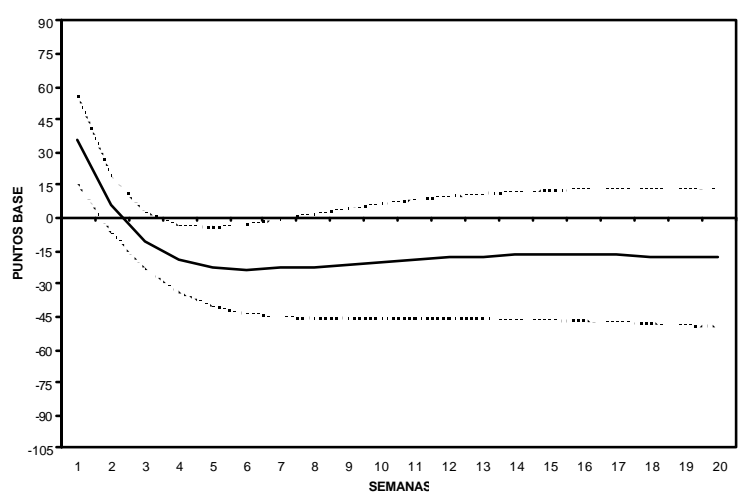

La Gráfica 11 presenta, para cada VAR estimado (entre paréntesis se señala el plazo de la tasa de interés utilizada), la respuesta de las expectativas de inflación

53 La magnitud exacta de una perturbación de una desviación estándar en el "corto" depende de cada VAR. Para el que incluye a la tasa de interés de CETES a 28 días es de 4.89 m.p., para los que incluyen a las tasas de interés de CETES a 91 y 182 días es de 4.91 m.p. y para el que incluye a la tasa de interés de CETES a 364 días es de 4.90 m.p. 
ante una perturbación restrictiva de la política monetaria54. Los resultados de la Gráfica anterior muestran que una política monetaria restrictiva "activa" reduce gradualmente y de manera permanente a las expectativas de inflación.

\section{Gráfica 11}
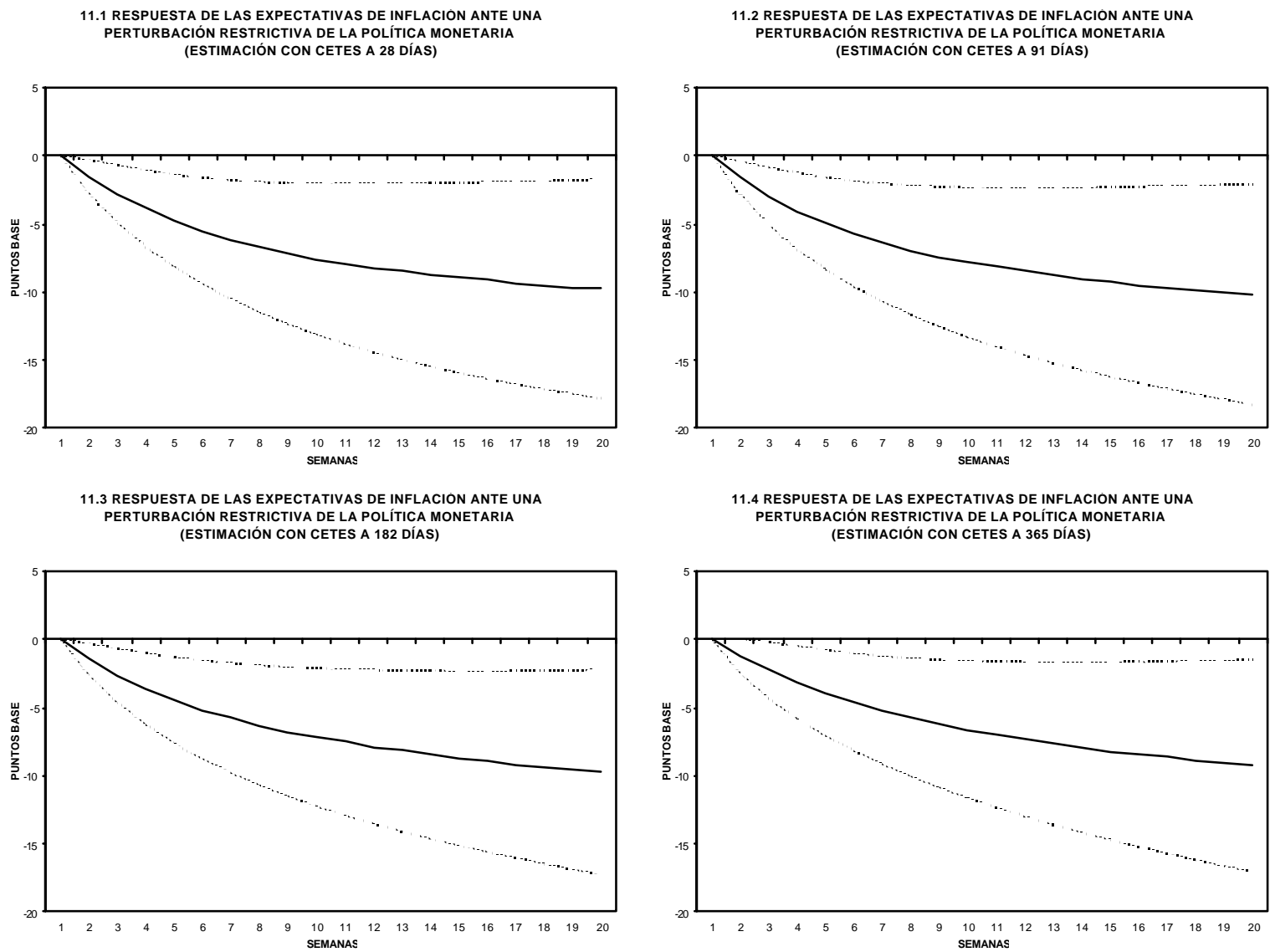

Con base en los resultados obtenidos hasta ahora, se puede decir que una política monetaria restrictiva "activa" no sólo incide de manera directa sobre el mercado de dinero a través de mayores tasas de interés, sino también en las expectativas de inflación. El comportamiento de las expectativas de inflación es congruente con el de las tasas de interés, donde después de aproximadamente tres semanas se observa un ajuste a la baja que es estadísticamente significativo, llegando a niveles inferiores a los que tenían antes del anuncio del "corto" (ver Gráfica 10). Es decir, la mejoría en las expectativas de inflación después de un anuncio restrictivo de política monetaria conduce, gradualmente, a menores tasas de interés. Lo anterior refleja que las expectativas de inflación de los agentes económicos reaccionan de acuerdo con las intenciones del Banco Central.

La Gráfica 12 presenta la respuesta de la curva de rendimiento ante una perturbación restrictiva (“activa”) en la variable de política monetaria de 20 m.p.,

54 Resultados similares se obtuvieron al colocar las expectativas de inflación después de la variable de política monetaria, "corto". Ver Grilli y Roubini (1995)) para una explicación sobre restricciones impuestas a las funciones impulso-respuesta de variables ordenadas antes de las variables sobre las que se analiza la perturbación. 
“controlando" por las variables de "reacción”. Al igual que en la Gráfica 9.1, en la Gráfica 12.1 la línea 1 corresponde al promedio de las tasas de interés para sus diferentes plazos durante el período del 25 de enero al 25 de abril de 2000. La línea 2 es la respuesta de la curva de rendimiento una semana después de la ampliación del "corto", estimada a partir de las funciones impulso-respuesta de la Gráfica 10, pero para una perturbación en el "corto" de 20 m.p. Finalmente, la línea 3 corresponde a la respuesta de la curva de rendimiento ante una política monetaria restrictiva después de dos semanas. Como se puede observar en la Gráfica 12.1, una política monetaria restrictiva "activa" tiene un efecto positivo sobre la curva de rendimiento, el cual va disminuyendo conforme pasa el tiempo y es mayor sobre las tasas de interés a menor plazo.

\section{Gráfica 12}
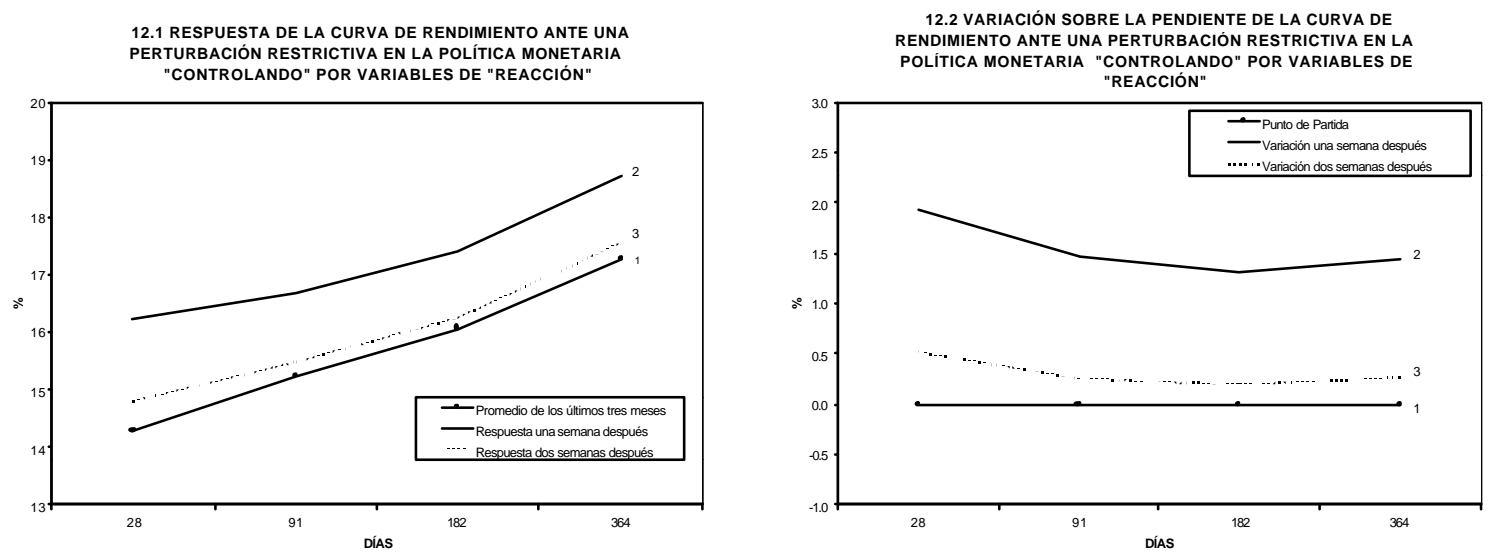

Para observar con mayor claridad el efecto de una política monetaria restrictiva "activa" sobre la pendiente de la curva de rendimiento, la Gráfica 12.2 presenta el alza inducida en las tasas de interés, a sus diferentes plazos, tras el anuncio de la restricción monetaria. Como se puede apreciar, las tasas de interés a plazos menores son más sensibles a la ampliación del "corto".

Adicionalmente, comparando las Gráficas 9 y 12 se puede observar que el efecto sobre la curva de rendimiento es menor al "controlar" por las variables de "reacción". Lo anterior se debe al hecho de que parte de la respuesta de las tasas de interés ante una perturbación restrictiva de la política monetaria se explica por el efecto ya descontado por el mercado. Esto se puede apreciar claramente en la Gráfica 13, donde se presenta la respuesta de la estructura de tasas de interés una y dos semanas después de la ampliación del "corto", sin "controlar" por las variables de "reacción" (línea 2) y "controlando" por las variables de "reacción" (línea 3). Ambas respuestas se obtuvieron para una ampliación en el "corto" de 20 m.p. Nuevamente, en las Gráficas 13.1 y 13.2, la línea 1 corresponde al promedio de las tasas de interés a diferentes plazos durante el período de tres meses ya referido, y las Gráficas 13.3 y 13.4 permiten apreciar con claridad el efecto de una restricción monetaria sobre la pendiente de la curva de rendimiento. 


\section{Gráfica 13}
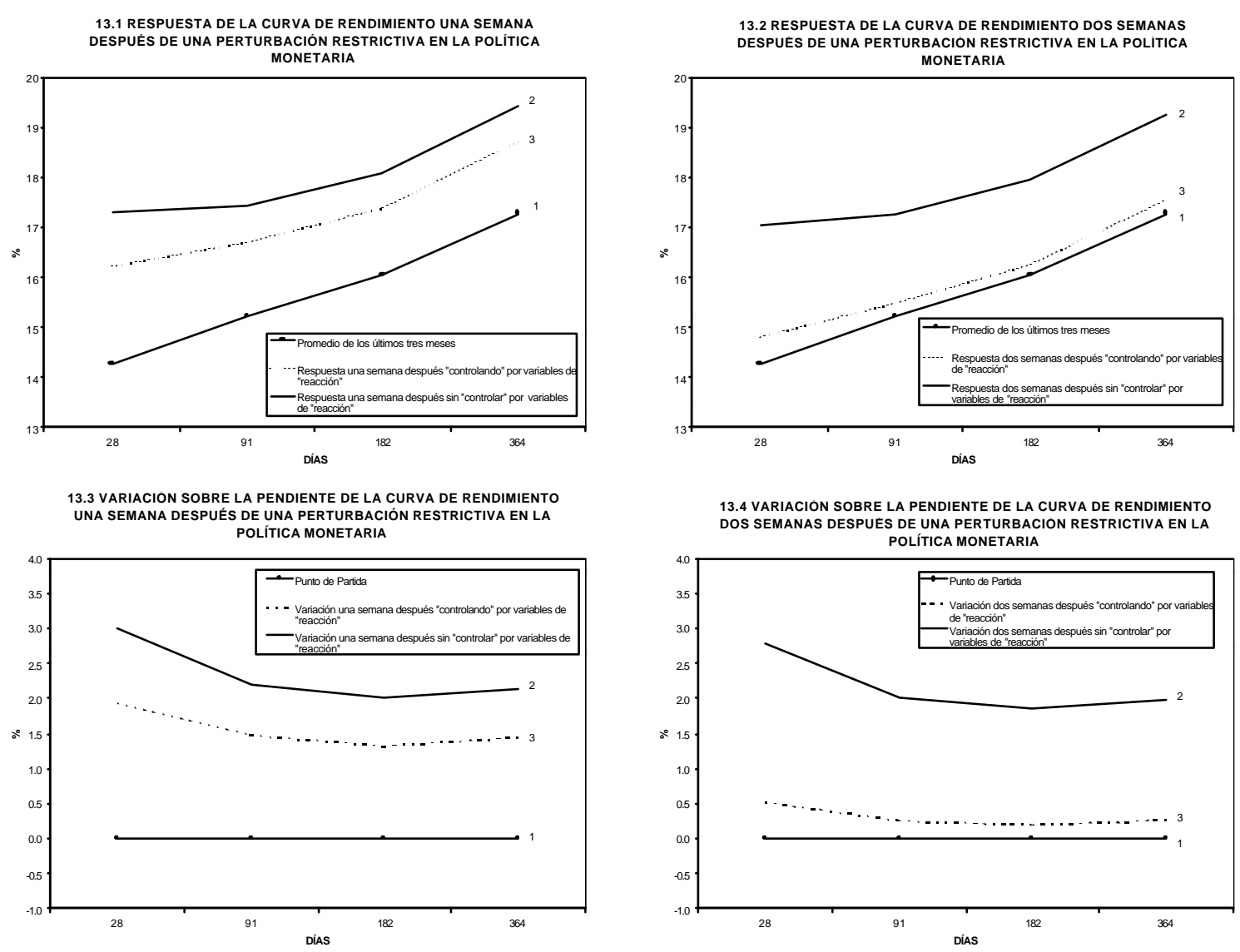

Como se puede observar en las Gráficas anteriores, aún "controlando" por el efecto "reacción", la política monetaria "activa" tiene efectos positivos y estadísticamente significativos sobre la estructura de tasas de interés. En otras palabras, hay evidencia que prueba el hecho de que la política monetaria en México impacta a las tasas de interés por encima del efecto "reacción". Lo anterior muestra que en los últimos años la política monetaria en México ha tenido un componente "activo".

Adicionalmente, se puede apreciar que el impacto de una restricción monetaria sobre la curva de rendimiento es menos duradero al "controlar" por las variables de "reacción". Una posible explicación de lo anterior puede ser la caída en las expectativas de inflación tras la ampliación del "corto". La referida disminución en las expectativas de inflación ocasiona que el impacto de una restricción monetaria sobre las tasas de interés sea transitorio e incluso que dichas tasas de interés disminuyan por debajo de su nivel inicial. 


\section{Conclusiones}

La efectividad de los bancos centrales para abatir la inflación, depende fundamentalmente del impacto que tengan sus acciones sobre los mercados financieros, lo que constituye el primer eslabón del mecanismo de transmisión de la política monetaria. Dichas acciones se canalizan a través de los llamados instrumentos de política monetaria. En el presente trabajo se describió la forma en que actualmente el Banco de México conduce su política monetaria. En particular se detalla el uso del objetivo de saldos acumulados o "corto" como instrumento de política monetaria.

Se presenta un análisis estático del impacto de cada "corto" adoptado desde 1998 sobre la estructura de tasas de interés, así como una descripción del contexto bajo el cual se anunciaron las modificaciones del "corto". A este respecto, en la mayoría de los casos, las tasas de interés a todos sus plazos se han incrementado en fechas posteriores a las modificaciones del "corto", para posteriormente disminuir gradualmente unas cuantas semanas después.

Las circunstancias bajo las cuales se ha restringido la postura de política monetaria durante los años de 1998, 1999 y 2000 (enero-abril) han sido muy variadas. Adicionalmente, si se considera que el régimen cambiario de libre flotación y la libre determinación de tasas de interés permiten al mercado ajustarse a las condiciones prevalecientes en la economía, no es fácil inferir qué parte de los ajustes observados en las tasas de interés responde exclusivamente al cambio de postura de política monetaria (política monetaria "activa) y cuál al ajuste de mercado (efecto "reacción").

En la sección III se analiza el impacto de cambios en el objetivo de saldos acumulados, en particular la adopción de un "corto", sobre la estructura de tasas de interés, descontando y sin descontar el efecto "reacción" sobre dichas tasas. Para lograr lo anterior se utilizó, la técnica de vectores autorregresivos. De éstas estimaciones se desprenden las siguientes conclusiones:

a) Una perturbación en la variable de política monetaria, tiene un efecto inicial positivo y estadísticamente significativo sobre las tasas de interés a sus diferentes plazos, aún descontando el efecto "reacción". Lo anterior implica que hay evidencia que prueba el hecho de que en los últimos años la política monetaria en México ha tenido un componente "activo".

b) Los resultados muestran que el componente "activo" de una restricción monetaria reduce gradualmente y de manera permanente las expectativas de inflación. El comportamiento de las expectativas de inflación es congruente con el de las tasas de interés, ya que después de aproximadamente tres semanas se observa un ajuste a la baja en dichas tasas, estadísticamente significativo, llegando a niveles inferiores a los que se tenían antes del anuncio del "corto". Lo anterior refleja que las expectativas de inflación de los agentes económicos reaccionan de acuerdo con las intenciones del Banco Central.

c) El efecto de una restricción monetaria es mayor sobre las tasas de interés de menor plazo y disminuye con el paso del tiempo.

d) Para las tasas de interés a todos sus plazos, el efecto de una ampliación en el 
“corto" es menor al "controlar" por las variables de "reacción”. Lo anterior, se debe al hecho de que parte de la respuesta de las tasas de interés ante cambios en la variable de política monetaria se explica por el efecto ya descontado por el mercado.

A pesar de que el Banco de México instrumenta su política monetaria a través del envío de señales, y no interfiriendo directamente en la determinación de las tasas de interés, la evidencia encontrada en este trabajo permite afirmar que la política monetaria en México ha tenido en los últimos años un componente "activo". Es decir, ha contribuido a inducir incrementos en las tasas de interés a sus diferentes plazos por encima del alza en tasas de interés que el mercado de dinero ha demandado. 
A N EX O 1

\begin{tabular}{|c|c|c|c|}
\hline \multicolumn{4}{|c|}{ Modificaciones en el Objetivo de Saldos Acumulados } \\
\hline Fecha de Cambio & $\begin{array}{l}\text { Objetivo de Saldos } \\
\text { Acumulados }\end{array}$ & Fecha de Cambio & $\begin{array}{l}\text { Objetivo de Saldos } \\
\text { Acumulados }\end{array}$ \\
\hline \multicolumn{2}{|c|}{1995} & \multicolumn{2}{|c|}{ 1996-2000 } \\
\hline 6 -sep-95 & 25 & 23-ene-96 & -5 \\
\hline 25-sep-95 & 20 & 25-ene-96 & -20 \\
\hline 30-oct-95 & 25 & 7-jun-96 & -31 \\
\hline 9-nov-95 & -200 & 21-jun-96 & -40 \\
\hline 13-nov-95 & -100 & 5-ago-96 & -30 \\
\hline 14-nov-95 & 100 & 19-ago-96 & 0 \\
\hline 15-nov-95 & 75 & 14-oct-96 & -20 \\
\hline 16-nov-95 & 65 & 26-nov-96 & 0 \\
\hline 17-nov-95 & 56 & 11-mar-98 & -20 \\
\hline 21-nov-95 & 25 & 25-jun-98 & -30 \\
\hline 23-nov-95 & 15 & 10-ago-98 & -50 \\
\hline 4-dic-95 & 0 & 17-ago-98 & -70 \\
\hline 8-dic-95 & -5 & 10-sep-98 & -100 \\
\hline 13-dic-95 & 0 & 30-nov-98 & -130 \\
\hline 14-dic-95 & 50 & 13-ene-99 & -160 \\
\hline 20-dic-95 & 25 & 18-ene-00 & -180 \\
\hline 28-dic-95 & 0 & & \\
\hline
\end{tabular}


A N EXO 2

PRUEBA DE RAÍZ UNITARIA DE DICKEY-FULLER AUMENTADA

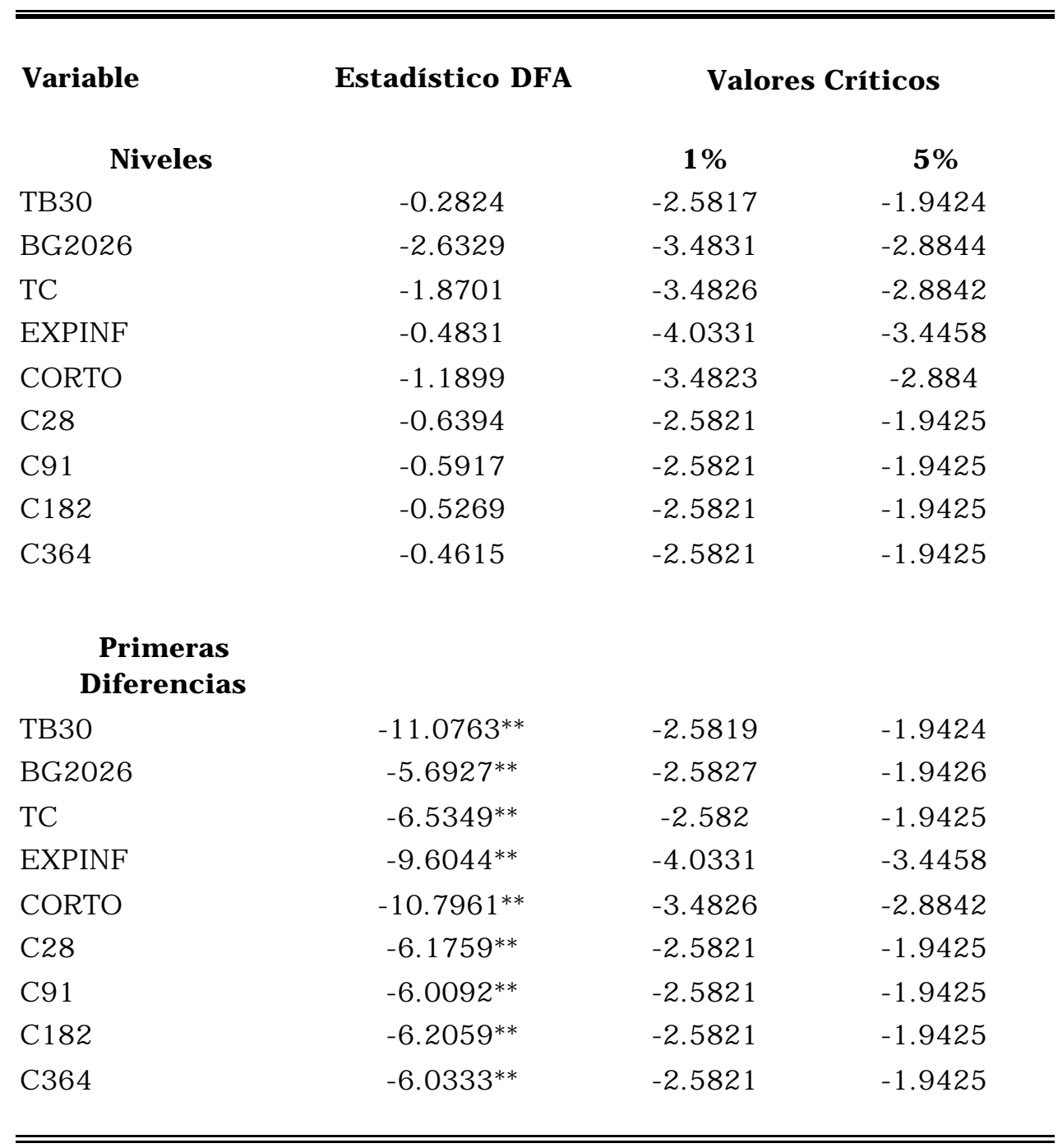

**(*) Significa rechazo de la hipótesis nula de raíz unitaria al 1\% (5\%) de significancia. 
PRUEBA DE COINTEGRACIÓN DE JOHANSEN

\begin{tabular}{|c|c|c|c|c|}
\hline \multirow{2}{*}{ Variables } & \multirow{2}{*}{$\begin{array}{l}\text { No. de Ecuaciones de } \\
\text { Cointegración (N) }\end{array}$} & \multirow{2}{*}{$\begin{array}{l}\text { Likelihood } \\
\text { Ratio }\end{array}$} & \multicolumn{2}{|c|}{ Valores Críticos } \\
\hline & & & $1 \%$ & $\mathbf{5 \%}$ \\
\hline \multirow[t]{2}{*}{ CORTO-C28 } & 0 & $18.4079^{*}$ & 20.04 & 15.41 \\
\hline & 1 & 2.2833 & 6.65 & 3.76 \\
\hline \multirow[t]{2}{*}{ CORTO-C91 } & 0 & $18.2211^{*}$ & 20.04 & 15.41 \\
\hline & 1 & 2.2388 & 6.65 & 3.76 \\
\hline \multirow[t]{2}{*}{ CORTO-C182 } & 0 & $16.8521^{*}$ & 20.04 & 15.41 \\
\hline & 1 & 2.366 & 6.65 & 3.76 \\
\hline \multirow[t]{2}{*}{ CORTO-C364 } & 0 & $17.5105^{*}$ & 20.04 & 15.41 \\
\hline & 1 & 2.55 & 6.65 & 3.76 \\
\hline \multirow[t]{6}{*}{ TB30-BG2026-TC-EXPINF-CORTO-C28 } & 0 & $118.7732^{* *}$ & 103.18 & 94.15 \\
\hline & 1 & 66.54 & 76.07 & 68.52 \\
\hline & 2 & 37.6166 & 54.46 & 47.21 \\
\hline & 3 & 21.8287 & 35.65 & 29.68 \\
\hline & 4 & 7.4672 & 20.04 & 15.41 \\
\hline & 5 & 0.0429 & 6.65 & 3.76 \\
\hline \multirow[t]{6}{*}{ TB30-BG2026-TC-EXPINF-CORTO-C91 } & 0 & $126.6165^{* *}$ & 103.18 & 94.15 \\
\hline & 1 & 67.1758 & 76.07 & 68.52 \\
\hline & 2 & 38.2554 & 54.46 & 47.21 \\
\hline & 3 & 18.7783 & 35.65 & 29.68 \\
\hline & 4 & 6.8129 & 20.04 & 15.41 \\
\hline & 5 & $3.50 \mathrm{E}-06$ & 6.65 & 3.76 \\
\hline \multirow[t]{6}{*}{ TB30-BG2026-TC-EXPINF-CORTO-C182 } & 0 & $129.2926^{* *}$ & 103.18 & 94.15 \\
\hline & 1 & $68.7362^{*}$ & 76.07 & 68.52 \\
\hline & 2 & 42.5004 & 54.46 & 47.21 \\
\hline & 3 & 17.159 & 35.65 & 29.68 \\
\hline & 4 & 6.5632 & 20.04 & 15.41 \\
\hline & 5 & 0.0009 & 6.65 & 3.76 \\
\hline \multirow[t]{6}{*}{ TB30-BG2026-TC-EXPINF-CORTO-C364 } & 0 & $130.0277^{* *}$ & 103.18 & 94.15 \\
\hline & 1 & $70.2444^{*}$ & 76.07 & 68.52 \\
\hline & 2 & 40.2551 & 54.46 & 47.21 \\
\hline & 3 & 16.6676 & 35.65 & 29.68 \\
\hline & 4 & 6.2101 & 20.04 & 15.41 \\
\hline & 5 & 0.0415 & 6.65 & 3.76 \\
\hline
\end{tabular}

**(*) Significa rechazo de la hipótesis nula de la existencia de $\mathrm{N}$ ecuaciones de cointegración al 1\% (5\%) de significancia. 


\section{Bibliografia}

Aguilar, Alejandro y V. Hugo Juan-Ramón, Determinantes de las Tasas de Interés a Corto Plazo en México: Efecto de las Señales del Banco Central, en Gaceta de Economía, La Política Monetaria en México, ITAM, Año 3, Número 5, Otoño 1997

Banco de México, Informe Anual, varios tomos.

Banco de México, Programa Monetario, varios tomos.

Banco de México, Boletín de Prensa, varios números.

Bernanke, Ben y Alan Blinder, The Federal Funds Rate and the Channels of Monetary Transmission, American Economic Review, Septiembre 1992, p. 901921.

Christiano, L.J., M. Eichenbaum and C. Evans, The Effects of Monetary Policy Shocks: Evidence from the Flow of Funds, Federal Reserve Bank of Chicago, working paper, No. 94-2, 1994 a.

Christiano, L.J., M. Eichenbaum and C. Evans, Identification and the effects of monetary policy shocks: Evidence from the flow of funds, Federal Reserve Bank of Chicago, working paper, No. 94-7, 1994b.

Christiano, L.J., M. Eichenbaum and C. Evans, Monetary Policy Shocks: What have we learned and to what end?, Northwestern University, Mimeo, 1997.

Edelberg Wendy y Marshall David. Monetary Policy Shocks and the Long-Term Interest Rates, Economic Perspectives, March/April 1996, Volume XX, Issue 2

Enders, Walter, Applied Econometric Time Series, Wiley, Primera Edición, USA, 1995, pp. 433

Gil Díaz, Francisco, La Política Monetaria y sus Canales de Transmisión en México, en Gaceta de Economía, La Política Monetaria en México, ITAM, Año 3, Número 5, Otoño 1997

Greenham Llorente, Laura Elena, Mecanismo de Transmisión de Política Monetaria, Tesis de Licenciatura, ITAM, 1997.

Hamilton, James D., Time Series Analysis, Princeton University Press, 1994.

Grilli, Vittorio y Nouriel Roubini, Liquidity and Exchange Rates: Puzzling Evidence from the G-7 countries, New York University Salomon Center, working paper, S-95-31, July 1995.

Juan-Ramón, Hugo, The Daily Conduct of Monetary Policy in Mexico, International Monetary Fund, Second (incomplete) draft, October 1996.

Leeper, Eric, Christopher Sims and Tao Zha, What Does Monetary Policy Do?, Brookins Papers on Economic Activity 2, 1996, p. 1-78. 
Martinez Trigueros, Lorenza, El Efecto de la Inflación en la Distribución del Ingreso, Banco de México, Documento de Investigación No. 9806, Diciembre 1998.

Mishkin, Frederick S., Symposium on the Monetary Transmission Mechanism, Journal of Economic Perspectives, Vol. 9, No. 4, Fall 1995, p. 3-10.

O’Dogherty, Pascual, La Instrumentación de la Politica Monetaria por el Banco Central, en Gaceta de Economía, La Política Monetaria en México, ITAM, Año 3, Número 5, Otoño 1997.

Pérez López, Alejandro y Moisés Schwartz, Inflación y Ciclos Económicos, Banco de México, Documento de Investigación No. 9904, 1999.

Schwartz Rosenthal, Moisés J., Consideraciones sobre la Instrumentación Práctica de la Política Monetaria, Banco de México, Documento de Investigación No. 9804, Octubre 1998. 